\title{
Inclusive dijet cross sections in neutral current deep inelastic scattering at HERA
}

\author{
The ZEUS Collaboration
}

H. Abramowicz ${ }^{45, b a}$, I. Abt ${ }^{35}$, L. Adamczyk ${ }^{13}$, M. Adamus ${ }^{54}$, R. Aggarwal $^{7}$, S. Antonelli ${ }^{4}$, P. Antonioli ${ }^{3}$, A. Antonov ${ }^{33}$, M. Arneodo ${ }^{50}$, V. Aushev ${ }^{26,27, \text { av }, \text { Y. Aushev }}{ }^{26,27, \text { av }}$, O. Bachynska ${ }^{15}$, A. Bamberger ${ }^{19}$, A.N. Barakbaev ${ }^{25}$, G. Barbagli ${ }^{17}$, G. Bari ${ }^{3}$, F. Barreiro ${ }^{30}$, D. Bartsch ${ }^{5}$, M. Basile ${ }^{4}$, O. Behnke ${ }^{15}$, J. Behr ${ }^{15}$, U. Behrens ${ }^{15}$, L. Bellagamba ${ }^{3}$, A. Bertolin ${ }^{39}$, S. Bhadra ${ }^{57}$, M. Bindi ${ }^{4}$, C. Blohm ${ }^{15}$, V. Bokhonov ${ }^{26,27}$, T. Bołd ${ }^{13}$, E.G. Boos ${ }^{25}$, K. Borras ${ }^{15}$, D. Boscherini ${ }^{3}$, D. Bot ${ }^{15}$, S.K. Boutle ${ }^{52}$, I. Brock ${ }^{5}$, E. Brownson ${ }^{56}$, R. Brugnera $^{40}$, N. Brümmer ${ }^{37}$, A. Bruni ${ }^{3}$, G. Bruni ${ }^{3}$, B. Brzozowska ${ }^{53}$, P.J. Bussey ${ }^{20}$, J.M. Butterworth ${ }^{52}$, B. Bylsma $^{37}$, A. Caldwell ${ }^{35}$, M. Capua $^{8}$, R. Carlin ${ }^{40}$, C.D. Catterall ${ }^{57}$, S. Chekanov ${ }^{1}$, J. Chwastowski ${ }^{12, z}$, J. Ciborowski ${ }^{53, \text { be }}$, R. Ciesielski ${ }^{15, \mathrm{ab}}$, L. Cifarelli ${ }^{4}$, F. Cindolo ${ }^{3}$, A. Contin $^{4}$, A.M. Cooper-Sarkar ${ }^{38}$, N. Coppola ${ }^{15}$,ac, M. Corradi $^{3}$, F. Corriveau ${ }^{31}$, M. Costa ${ }^{49}$, G. D’Agostini ${ }^{43}$, F. Dal Corso ${ }^{39}$, J. del Peso ${ }^{30}$, R.K. Dementiev ${ }^{34}$, S. De Pasquale $^{4, v}$, M. Derrick ${ }^{1}$, R.C.E. Devenish ${ }^{38}$, D. Dobur ${ }^{19, \text { ao }}$, B.A. Dolgoshein ${ }^{33}$, G. Dolinska ${ }^{26,27}$, A.T. Doyle ${ }^{20}$, V. Drugakov ${ }^{16}$, L.S. Durkin ${ }^{37}$, S. Dusini ${ }^{39}$, Y. Eisenberg ${ }^{55}$, P.F. Ermolov ${ }^{34, a u}$, A. Eskreys ${ }^{12}$, S. Fang ${ }^{15, \text { ad }, \text { S. Fazio }^{8} \text {, J. Ferrando }}{ }^{38}$,

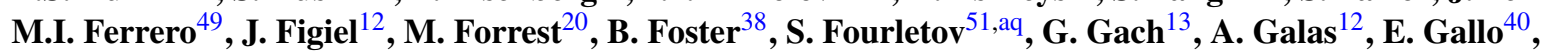

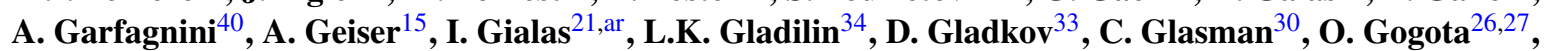
Yu.A. Golubkov ${ }^{34}$, P. Göttlicher ${ }^{15, \text { ae }}$, I. Grabowska-Bołd ${ }^{13}$, J. Grebenyuk ${ }^{15}$, I. Gregor ${ }^{15}$, G. Grigorescu $^{36}$,

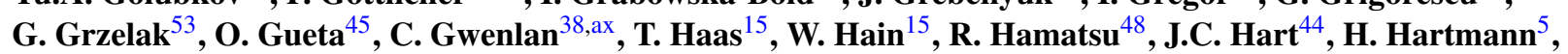

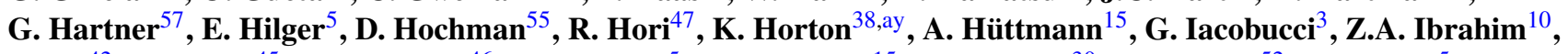
Y. Iga ${ }^{42}$, R. Ingbir ${ }^{45}$, M. Ishitsuka ${ }^{46}$, H.-P. Jakob ${ }^{5}$, F. Januschek ${ }^{15}$, M. Jimenez ${ }^{30}$, T.W. Jones ${ }^{52}$, M. Jüngst ${ }^{5}$, I. Kadenko ${ }^{26,27}$, B. Kahle ${ }^{15}$, B. Kamaluddin ${ }^{10, a u}$, S. Kananov ${ }^{45}$, T. Kanno ${ }^{46}$, U. Karshon ${ }^{55}$, F. Karstens ${ }^{19, \text { ap }}$, I.I. Katkov ${ }^{15, \text { af }}$, M. Kaur ${ }^{7}$, P. Kaur ${ }^{7, x}$, A. Keramidas ${ }^{36}$, L.A. Khein ${ }^{34}$, J.Y. Kim ${ }^{9}$, D. Kisielewska ${ }^{13}$, S. Kitamura ${ }^{48, b c}$, R. Klanner ${ }^{22}$, U. Klein ${ }^{15, \text { ag }}$, E. Koffeman ${ }^{36}$, P. Kooijman ${ }^{36}$, Ie. Korol ${ }^{26,27}$, I.A. Korzhavina ${ }^{34}$, A. Kotański ${ }^{14, a a}$, U. Kötz ${ }^{15}$, H. Kowalski ${ }^{15}$, P. Kulinski ${ }^{53}$, O. Kuprash ${ }^{26,27, \text { aw }}$, M. Kuze ${ }^{46}$, A. Lee ${ }^{37}$, B.B. Levchenko ${ }^{34}$, A. Levy ${ }^{45, a}$, V. Libov ${ }^{15}$, S. Limentani ${ }^{40}$, T.Y. Ling ${ }^{37}$, M. Lisovyi ${ }^{15}$, E. Lobodzinska ${ }^{15}$, W. Lohmann ${ }^{16}$, B. Löhr ${ }^{15}$, E. Lohrmann $^{22}$, J.H. Loizides ${ }^{52}$, K.R. Long $^{23}$, A. Longhin ${ }^{39}$, D. Lontkovskyi ${ }^{26,27, \text { aw }}$, O.Yu. Lukina ${ }^{34}$, P. Lużniak ${ }^{53, b f}$, J. Maeda ${ }^{46, b b}$, S. Magill $^{1}$, I. Makarenko ${ }^{26,27, \text { aw }}$, J. Malka ${ }^{53, \text { bf }}$, R. Mankel ${ }^{15}$, A. Margotti $^{3}$, G. Marini ${ }^{43}$, J.F. Martin ${ }^{51}$, A. Mastroberardino ${ }^{8}$, M.C.K. Mattingly ${ }^{2}$, I.-A. Melzer-Pellmann ${ }^{15}$, S. Miglioranzi ${ }^{15}$, ah, F. Mohamad Idris ${ }^{10}$,

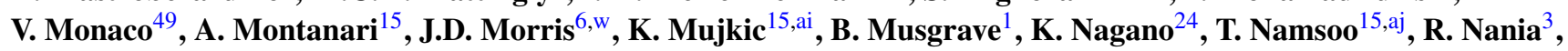
D. Nicholass ${ }^{1, \mathrm{u}}$, A. Nigro $^{43}$, Y. Ning ${ }^{11}$, U. Noor ${ }^{57}$, D. Notz $^{15}$, R.J. Nowak ${ }^{53}$, A.E. Nuncio-Quiroz ${ }^{5}$, B.Y. Oh ${ }^{41}$, N. Okazaki ${ }^{47}$, K. Oliver $^{38}$, K. Olkiewicz ${ }^{12}$, Yu. Onishchuk ${ }^{26,27}$, K. Papageorgiu ${ }^{21}$, A. Parenti ${ }^{15}$, E. Paul ${ }^{5}$, J.M. Pawlak ${ }^{53}$, B. Pawlik ${ }^{12}$, P. Pelfer ${ }^{18}$, A. Pellegrino ${ }^{36}$, W. Perlanski ${ }^{53, b f}$, H. Perrey ${ }^{22}$, K. Piotrzkowski $^{29}$, P. Plucinski ${ }^{54, b g}$, N.S. Pokrovskiy ${ }^{25}$, A. Polini ${ }^{3}$, A.S. Proskuryakov ${ }^{34}$, M. Przybycień ${ }^{13}$, A. Raval $^{15}$, D.D. Reeder $^{56}$,

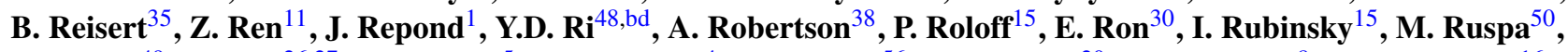
R. Sacchi ${ }^{49}$, A. Salii ${ }^{26,27}$, U. Samson ${ }^{5}$, G. Sartorelli ${ }^{4}$, A.A. Savin ${ }^{56}$, D.H. Saxon ${ }^{20}$, M. Schioppa ${ }^{8}$, S. Schlenstedt ${ }^{16}$, P. Schleper ${ }^{22}$, W.B. Schmidke ${ }^{35}$, U. Schneekloth ${ }^{15}$, V. Schönberg ${ }^{5}$, T. Schörner-Sadenius ${ }^{15}$, J. Schwartz ${ }^{31}$, F. Sciulli ${ }^{11}$, L.M. Shcheglova ${ }^{34}$, R. Shehzadi ${ }^{5}$, S. Shimizu ${ }^{47, \text { ah, } \text { I. Singh }^{7, x} \text {, I.O. Skillicorn }}{ }^{20}$, W. Słomiński ${ }^{14}$, W.H. Smith ${ }^{56}$, V. Sola ${ }^{49}$, A. Solano ${ }^{49}$, D. Son ${ }^{28}$, V. Sosnovtsev ${ }^{33}$, A. Spiridonov ${ }^{15, \text { ak }}$, H. Stadie ${ }^{22}$, L. Stanco ${ }^{39}$, A. Stern ${ }^{45}$, T.P. Stewart ${ }^{51}$, A. Stifutkin ${ }^{33}$, P. Stopa ${ }^{12}$, S. Suchkov ${ }^{33}$, G. Susinno ${ }^{8}$, L. Suszycki ${ }^{13}$, J. Sztuk-Dambietz ${ }^{22}$,

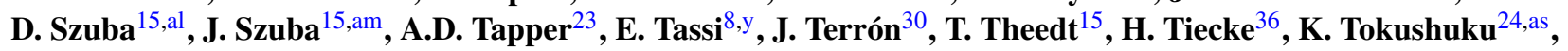
O. Tomalak $^{26,27}$, J. Tomaszewska ${ }^{15, \text { an }}$, T. Tsurugai ${ }^{32}$, M. Turcato ${ }^{22}$, T. Tymieniecka ${ }^{54, \text { th }}$, C. Uribe-Estrada ${ }^{30}$, M. Vázquez ${ }^{36, a h}$, A. Verbytskyi ${ }^{15}$, O. Viazlo ${ }^{26,27}$, N.N. Vlasov ${ }^{19, \text { aq }}$, O. Volynets $^{26,27}$, R. Walczak ${ }^{38}$, W.A.T. Wan Abdullah $^{10}$, J.J. Whitmore ${ }^{41, a z}$, J. Whyte ${ }^{57}$, L. Wiggers ${ }^{36}$, M. Wing ${ }^{52}$, M. Wlasenko ${ }^{5}$, G. Wolf ${ }^{15}$, H. Wolfe ${ }^{56}$, K. Wrona ${ }^{15}$, A.G. Yagües-Molina ${ }^{15}$, S. Yamada ${ }^{24}$, Y. Yamazaki ${ }^{24, \text { at }}$, R. Yoshida ${ }^{1}$, C. Youngman ${ }^{15}$, A.F. Żarnecki ${ }^{53}$, L. Zawiejski ${ }^{12}$, O. Zenaiev ${ }^{26,27}$, W. Zeuner ${ }^{15, \text { ah }}$, B.O. Zhautykov ${ }^{25}$, N. Zhmak ${ }^{26,27, \text { av }}$, C. Zhou ${ }^{31}$, A. Zichichi ${ }^{4}$, M. Zolko ${ }^{26,27}$, D.S. Zotkin ${ }^{34}$, Z. Zulkapli ${ }^{10}$ 
${ }^{1}$ Argonne National Laboratory, Argonne, IL 60439-4815, USA ${ }^{b}$

${ }^{2}$ Andrews University, Berrien Springs, MI 49104-0380 USA

${ }^{3}$ INFN Bologna, Bologna, Italy ${ }^{\mathrm{c}}$

${ }^{4}$ University and INFN Bologna, Bologna, Italy ${ }^{\mathrm{c}}$

${ }^{5}$ Physikalisches Institut der Universität Bonn, Bonn, Germany ${ }^{\mathrm{d}}$

${ }^{6}$ H.H. Wills Physics Laboratory, University of Bristol, Bristol, $\mathrm{UK}^{\mathrm{e}}$

${ }^{7}$ Department of Physics, Panjab University, Chandigarh, India

${ }^{8}$ Physics Department and INFN, Calabria University, Cosenza, Italy ${ }^{\mathrm{c}}$

${ }^{9}$ Institute for Universe and Elementary Particles, Chonnam National University, Kwangju, South Korea

${ }^{10}$ Jabatan Fizik, Universiti Malaya, 50603, Kuala Lumpur, Malaysia ${ }^{\mathrm{f}}$

${ }^{11}$ Nevis Laboratories, Columbia University, Irvington on Hudson, NY 10027, USA ${ }^{g}$

${ }^{12}$ The Henryk Niewodniczanski Institute of Nuclear Physics, Polish Academy of Sciences, Cracow, Poland ${ }^{\text {h }}$

${ }^{13}$ Faculty of Physics and Applied Computer Science, AGH-University of Science and Technology, Cracow, Poland ${ }^{\mathrm{i}}$

${ }^{14}$ Department of Physics, Jagellonian University, Cracow, Poland

${ }^{15}$ Deutsches Elektronen-Synchrotron DESY, Hamburg, Germany

${ }^{16}$ Deutsches Elektronen-Synchrotron DESY, Zeuthen, Germany

${ }^{17}$ INFN Florence, Florence, Italy ${ }^{\mathrm{c}}$

${ }^{18}$ University and INFN Florence, Florence, Italy ${ }^{\mathrm{c}}$

${ }^{19}$ Fakultät für Physik der Universität Freiburg i.Br., Freiburg i.Br., Germany

${ }^{20}$ School of Physics and Astronomy, University of Glasgow, Glasgow, UK ${ }^{\mathrm{e}}$

${ }^{21}$ Department of Engineering in Management and Finance, Univ. of the Aegean, Chios, Greece

${ }^{22}$ Institute of Experimental Physics, Hamburg University, Hamburg, Germany ${ }^{\mathrm{j}}$

${ }^{23}$ High Energy Nuclear Physics Group, Imperial College London, London, UK ${ }^{\mathrm{e}}$

${ }^{24}$ Institute of Particle and Nuclear Studies, KEK, Tsukuba, Japan ${ }^{\mathrm{k}}$

${ }^{25}$ Institute of Physics and Technology of Ministry of Education and Science of Kazakhstan, Almaty, Kazakhstan

${ }^{26}$ Institute for Nuclear Research, National Academy of Sciences, Kiev, Ukraine

${ }^{27}$ Kiev National University, Kiev, Ukraine

${ }^{28}$ Center for High Energy Physics, Kyungpook National University, Daegu, South Korea ${ }^{1}$

${ }^{29}$ Institut de Physique Nucléaire, Université Catholique de Louvain, Louvain-la-Neuve, Belgium ${ }^{\mathrm{m}}$

${ }^{30}$ Departamento de Física Teórica, Universidad Autónoma de Madrid, Madrid, Spain ${ }^{\mathrm{n}}$

${ }^{31}$ Department of Physics, McGill University, Montréal, Québec, Canada H3A 2T8 ${ }^{\circ}$

${ }^{32}$ Faculty of General Education, Meiji Gakuin University, Yokohama, Japan ${ }^{\mathrm{k}}$

${ }^{33}$ Moscow Engineering Physics Institute, Moscow, Russia ${ }^{p}$

${ }^{34}$ Institute of Nuclear Physics, Moscow State University, Moscow, Russia ${ }^{\mathrm{q}}$

${ }^{35}$ Max-Planck-Institut für Physik, München, Germany

${ }^{36}$ NIKHEF and University of Amsterdam, Amsterdam, Netherlands ${ }^{\mathrm{r}}$

${ }^{37}$ Physics Department, Ohio State University, Columbus, OH 43210, USA ${ }^{b}$

${ }^{38}$ Department of Physics, University of Oxford, Oxford, UK ${ }^{\mathrm{e}}$

${ }^{39}$ INFN Padova, Padova, Italy ${ }^{\mathrm{c}}$

${ }^{40}$ Dipartimento di Fisica dell' Università and INFN, Padova, Italy ${ }^{\mathrm{c}}$

${ }^{41}$ Department of Physics, Pennsylvania State University, University Park, PA 16802, USA ${ }^{\mathrm{g}}$

${ }^{42}$ Polytechnic University, Sagamihara, Japan ${ }^{\mathrm{k}}$

${ }^{43}$ Dipartimento di Fisica, Università 'La Sapienza' and INFN, Rome, Italy ${ }^{\mathrm{c}}$

${ }^{44}$ Rutherford Appleton Laboratory, Chilton, Didcot, Oxon, UK ${ }^{\mathrm{e}}$

${ }^{45}$ Raymond and Beverly Sackler Faculty of Exact Sciences, School of Physics, Tel Aviv University, Tel Aviv, Israel ${ }^{\mathrm{s}}$

${ }^{46}$ Department of Physics, Tokyo Institute of Technology, Tokyo, Japan ${ }^{\mathrm{k}}$

${ }^{47}$ Department of Physics, University of Tokyo, Tokyo, Japan ${ }^{\mathrm{k}}$

${ }^{48}$ Department of Physics, Tokyo Metropolitan University, Tokyo, Japan ${ }^{\mathrm{k}}$

${ }^{49}$ Università di Torino and INFN, Torino, Italyc

${ }^{50}$ Università del Piemonte Orientale, Novara, and INFN, Torino, Italy ${ }^{\mathrm{c}}$

${ }^{51}$ Department of Physics, University of Toronto, Toronto, Ontario, Canada, M5S 1A7 ${ }^{\circ}$

${ }^{52}$ Physics and Astronomy Department, University College London, London, $\mathrm{UK}^{\mathrm{e}}$

${ }^{53}$ Institute of Experimental Physics, Warsaw University, Warsaw, Poland

${ }^{54}$ Institute for Nuclear Studies, Warsaw, Poland

${ }_{56}^{55}$ Department of Particle Physics, Weizmann Institute, Rehovot, Israel ${ }^{t}$

${ }^{56}$ Department of Physics, University of Wisconsin, Madison, WI 53706, USA ${ }^{\mathrm{b}}$

${ }^{57}$ Department of Physics, York University, Toronto, Ontario, Canada M3J 1P3 ${ }^{\circ}$

Received: 28 October 2010 / Published online: 24 November 2010

(C) The Author(s) 2010. This article is published with open access at Springerlink.com

\footnotetext{
a e-mail: levy@alzt.tau.ac.il

${ }^{\mathrm{b}}$ Supported by the US Department of Energy.
}

${ }^{\mathrm{c}}$ Supported by the Italian National Institute for Nuclear Physics (INFN). 
Abstract Single- and double-differential inclusive dijet cross sections in neutral current deep inelastic ep scattering have been measured with the ZEUS detector using an integrated luminosity of $374 \mathrm{pb}^{-1}$. The measurement was performed at large values of the photon virtuality, $Q^{2}$, between 125 and $20000 \mathrm{GeV}^{2}$. The jets were reconstructed with the $k_{T}$ cluster algorithm in the Breit reference frame and selected by requiring their transverse energies in the Breit frame, $E_{T, \mathrm{~B}}^{\mathrm{jet}}$, to be larger than $8 \mathrm{GeV}$. In addition, the invariant mass of the dijet system, $M_{\mathrm{jj}}$, was required to be

${ }^{\mathrm{d}}$ Supported by the German Federal Ministry for Education and Research (BMBF), under contract No. 05 H09PDF.

${ }^{\text {e }}$ Supported by the Science and Technology Facilities Council, UK.

${ }^{\text {f }}$ Supported by an FRGS grant from the Malaysian government.

${ }^{\mathrm{g}}$ Supported by the US National Science Foundation. Any opinion, findings and conclusions or recommendations expressed in this material are those of the authors and do not necessarily reflect the views of the National Science Foundation.

${ }^{\mathrm{h}}$ Supported by the Polish Ministry of Science and Higher Education as a scientific project No. DPN/N188/DESY/2009.

${ }^{\text {i }}$ Supported by the Polish Ministry of Science and Higher Education as a scientific project (2009-2010).

${ }^{\mathrm{j}}$ Supported by the German Federal Ministry for Education and Research (BMBF), under contract No. 05h09GUF, and the SFB 676 of the Deutsche Forschungsgemeinschaft (DFG).

${ }^{\mathrm{k}}$ Supported by the Japanese Ministry of Education, Culture, Sports, Science and Technology (MEXT) and its grants for Scientific Research.

${ }^{\text {l}}$ Supported by the Korean Ministry of Education and Korea Science and Engineering Foundation.

${ }^{\mathrm{m}}$ Supported by FNRS and its associated funds (IISN and FRIA) and by an Inter-University Attraction Poles Programme subsidised by the Belgian Federal Science Policy Office.

${ }^{\mathrm{n}}$ Supported by the Spanish Ministry of Education and Science through funds provided by CICYT.

${ }^{\circ}$ Supported by the Natural Sciences and Engineering Research Council of Canada (NSERC).

pPartially Supported by the German Federal Ministry for Education and Research (BMBF).

${ }^{\mathrm{q}}$ Supported by RF Presidential grant N 41-42.2010.2 for the Leading Scientific Schools and by the Russian Ministry of Education and Science through its grant for Scientific Research on High Energy Physics.

${ }^{\mathrm{r}}$ Supported by the Netherlands Foundation for Research on Matter (FOM).

${ }^{\mathrm{s}}$ Supported by the Israel Science Foundation.

${ }^{\text {t}}$ Supported in part by the MINERVA Gesellschaft für Forschung GmbH, the Israel Science Foundation (grant No. 293/02-11.2) and the US-Israel Binational Science Foundation.

uAlso affiliated with University College London, United Kingdom.

${ }^{\mathrm{v}}$ Now at University of Salerno, Italy.

${ }^{w}$ Now at Queen Mary University of London, United Kingdom.

${ }^{x}$ Also working at Max Planck Institute, Munich, Germany.

${ }^{\mathrm{y}}$ Also Senior Alexander von Humboldt Research Fellow at Hamburg University, Institute of Experimental Physics, Hamburg, Germany. greater than $20 \mathrm{GeV}$. The cross sections are described by the predictions of next-to-leading-order QCD.

\section{Introduction}

Measurements of jet cross sections are a well established tool for QCD studies and have been performed for many different observables at HERA [1-17]. For jet analyses in neutral current (NC) deep inelastic scattering (DIS), the Breit reference frame $[18,19]$ is preferred, since it provides a maximal separation between the products of the beam frag-

${ }^{\mathrm{z}}$ Also at Cracow University of Technology, Faculty of Physics, Mathemathics and Applied Computer Science, Poland.

${ }^{\text {aa }}$ Supported by the research grant No. 1 P03B 04529 (2005-2008).

${ }^{\mathrm{ab}}$ Now at Rockefeller University, New York, NY 10065, USA.

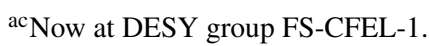

${ }^{a d}$ Now at Institute of High Energy Physics, Beijing, China.

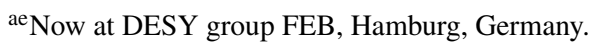

${ }^{a f}$ Also at Moscow State University, Russia.

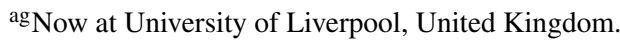

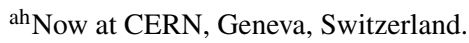

ai Also affiliated with University College London, UK.

ajNow at Goldman Sachs, London, UK.

${ }^{\mathrm{ak}}$ Also at Institute of Theoretical and Experimental Physics, Moscow, Russia.

${ }^{\text {al }}$ Also at INP, Cracow, Poland.

${ }^{a m}$ Also at FPACS, AGH-UST, Cracow, Poland.

${ }^{\text {an }}$ Partially supported by Warsaw University, Poland.

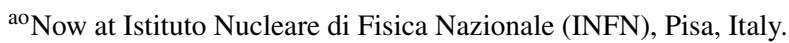

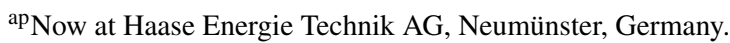

${ }^{a q}$ Now at Department of Physics, University of Bonn, Germany.

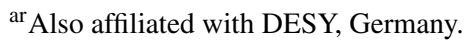

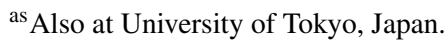

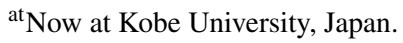

au Deceased.

${ }^{\text {av }}$ Supported by DESY, Germany.

${ }^{\text {aw }}$ Supported by the Bogolyubov Institute for Theoretical Physics of the National Academy of Sciences, Ukraine.

${ }^{a x}$ STFC Advanced Fellow.

${ }^{\text {ay }}$ Nee Korcsak-Gorzo.

${ }^{\mathrm{az}}$ This material was based on work supported by the National Science Foundation, while working at the Foundation.

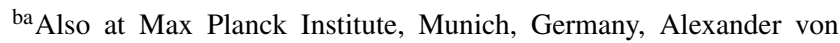
Humboldt Research Award.

${ }^{\mathrm{bb}}$ Now at Tokyo Metropolitan University, Japan.

${ }^{\mathrm{bc}}$ Now at Nihon Institute of Medical Science, Japan.

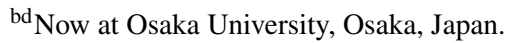

be Also at Łódź University, Poland.

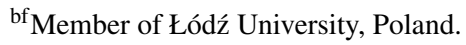

bg Now at Lund University, Lund, Sweden.

bh Also at University of Podlasie, Siedlce, Poland. 
mentation and the hard jets. In this frame, the exchanged space-like virtual boson has 3-momentum $\boldsymbol{q}=(0,0,-Q)$ and is collinear with the incoming parton. While retaining hard QCD processes at leading order in the strong coupling constant $\alpha_{s}$, the contribution from the parton-model process can be suppressed by requiring the production of jets with high transverse energy in the Breit frame. Therefore, measurements of jet cross sections in the Breit frame are directly sensitive to hard QCD processes, allowing tests of perturbative QCD (pQCD), of the factorisation ansatz and of the parton distribution functions (PDFs) of the proton.

At large boson virtualities, $Q^{2}$, the experimental and theoretical systematic uncertainties are small and, thus, use of the large HERA data sample can provide powerful physical constraints. Jet cross-section data from the high- $Q^{2}$ region have been included in the ZEUS PDF fit, thereby significantly reducing the uncertainty on the gluon density in the medium- to high- $x$ region [20].

Measurements of dijet production in DIS at HERA have so far been performed with either smaller data sets $[1,2,7]$ or normalised to the inclusive NC DIS cross section [9]. In this paper, measurements of inclusive dijet production at large values of $Q^{2}$ are presented using an integrated luminosity of $374 \mathrm{pb}^{-1}$. Here, differential dijet cross sections as a function of $Q^{2}$, of the mean jet transverse energy of the dijet system in the Breit frame, $\overline{E_{T, B}^{\mathrm{jet}}}$, of the dijet invariant mass, $M_{\mathrm{jj}}$, of the half-difference of the jet pseudorapidities in the Breit frame, $\eta^{*}=\left|\eta_{\mathrm{B}}^{\text {jet} 1}-\eta_{\mathrm{B}}^{\text {jet2 }}\right| / 2$, of the fraction of the proton momentum taken by the interacting parton, $\xi=x_{\mathrm{Bj}}\left(1+\left(M_{\mathrm{jj}}\right)^{2} / Q^{2}\right)$, and of $x_{\mathrm{Bj}}$ are presented. Here, $x_{\mathrm{Bj}}$ is the Bjorken scaling variable that defines, for the parton-model process, the fraction of the proton momentum carried by the struck massless parton. Measurements of the dijet cross section as a function of $\xi$ and $\overline{E_{T, \mathrm{~B}}^{\text {jet }}}$ are also shown for different regions of $Q^{2}$.

The measured single- and double-differential cross sections are compared with next-to-leading-order (NLO) QCD calculations.

\section{Experimental set-up}

A detailed description of the ZEUS detector can be found elsewhere [21]. A brief outline of the components that are most relevant for this analysis is given below.

Charged particles were tracked in the central tracking detector (CTD) [22-24], which operated in a magnetic field of $1.43 \mathrm{~T}$ provided by a thin superconducting solenoid. The CTD consisted of 72 cylindrical drift-chamber layers, organised in nine superlayers covering the polar-angle region $15^{\circ}<\theta<164^{\circ}$. For data taken during the years 1998 to 2000 , tracks were reconstructed using the CTD only. Starting from the year 2004, the CTD and a silicon microvertex detector (MVD) [25], installed between the beam-pipe and the inner radius of the CTD, were used.

The high-resolution uranium-scintillator calorimeter (CAL) [26-29] covered $99.7 \%$ of the total solid angle and consisted of three parts: the forward (FCAL), the barrel (BCAL) and the rear (RCAL) calorimeters. Each part was subdivided transversely into towers and longitudinally into one electromagnetic section (EMC) and either one (in RCAL) or two (in BCAL and FCAL) hadronic sections (HAC). The smallest subdivision of the calorimeter is called a cell. Under test-beam conditions, the CAL single-particle relative energy resolutions were $\sigma(E) / E=0.18 / \sqrt{E}$ for (anti-)electrons and $\sigma(E) / E=0.35 / \sqrt{E}$ for hadrons, with $E$ in $\mathrm{GeV}$.

The luminosity was measured using the Bethe-Heitler reaction $e p \rightarrow e \gamma p$ by the luminosity detector which consisted of a lead-scintillator [30-32] calorimeter and, in the 20042007 running period, an independent magnetic spectrometer [33].

The electron ${ }^{1}$ beam in HERA was naturally transversely polarised through the Sokolov-Ternov effect $[34,35]$. The characteristic polarisation build-up time for the HERA accelerator was approximately 40 minutes. Starting from the year 2004, spin rotators on either side of the ZEUS detector changed the transverse polarisation of the beam into longitudinal polarisation and back again. The electron beam polarisation was measured using two independent polarimeters, the transverse polarimeter (TPOL) [36] and the longitudinal polarimeter (LPOL) [37]. Both devices exploited the spindependent cross section for Compton scattering of circularly polarised photons off electrons to measure the beam polarisation. The luminosity and polarisation measurements were made over time intervals that were much shorter than the polarisation build-up time.

\section{Event selection and reconstruction}

The data used in this analysis were collected during the periods 1998-2000 and 2004-2007, when HERA operated with protons of energy $E_{p}=920 \mathrm{GeV}$ and electrons or positrons of energy $E_{e}=27.5 \mathrm{GeV}$, and correspond to an integrated luminosity of $203 \mathrm{pb}^{-1}$ for the electron data and $171 \mathrm{pb}^{-1}$ for the positron data. The mean luminosity-weighted average polarisation of the data was -0.03 .

A three-level trigger system was used to select events online $[21,38,39]$. At the third level, NC DIS events were accepted on the basis of the identification of a scatteredelectron candidate using localised energy depositions in the

\footnotetext{
${ }^{1}$ Here and in the following, the term "electron" denotes generically both the electron and the positron unless otherwise stated.
} 
CAL. At the second level, charged-particle tracks were reconstructed online by using the ZEUS global tracking trigger [40], which combined information from the CTD and MVD. These online tracks were used to reconstruct the interaction vertex and reject non-ep background. At the first level, only coarse calorimeter and tracking information was available. Events were selected using criteria based on the energy and transverse energy measured in the CAL. Starting from the year 2004, additional tracking requirements were introduced to adapt the trigger rates to the higher instantaneous luminosity.

Events were selected offline using criteria which were slightly changed with respect to those used in the previous ZEUS dijet measurement [7]. These changes reflect the new phase-space definition of the measurements adopted here. The main steps of the selection are briefly listed below.

The scattered-electron candidate was identified from the pattern of energy deposits in the CAL [41, 42]. The energy, $E_{e}^{\prime}$, the polar ${ }^{2}$ angle, $\theta_{e}$, and the azimuthal angle, $\phi_{e}$, of the electron candidate were determined from the CAL measurements. For events in which the electron was found inside the CTD acceptance, the angles $\theta_{e}$ and $\phi_{e}$ were reconstructed from the associated electron track. The photon virtuality $Q^{2}$ and the Bjorken scaling variable $x_{\mathrm{Bj}}$ were reconstructed using the double angle (DA) method [43, 44]. The inelasticity variable $y$ was determined from the condition $y=Q^{2} / x_{\mathrm{Bj}} s$, where $s$ is the square of the centre-of-mass energy.

\subsection{Inclusive event selection}

The phase space of the measurement was $125<Q^{2}<$ $20000 \mathrm{GeV}^{2}$ and $0.2<y<0.6$.

Events were selected if:

- An electron candidate of energy $E_{e}^{\prime}>10 \mathrm{GeV}$ was found. This requirement ensured a high and well understood electron-finding efficiency and suppressed background from photoproduction events in which the scattered electron escapes down the rear beampipe.

- The total energy not associated with the electron candidate within a cone of radius 0.7 units in the pseudorapidity-azimuth $(\eta-\varphi)$ plane around the electron direction was less than $10 \%$ of the electron energy. This condition removed photoproduction and DIS events in which part of a jet was falsely identified as the scattered electron.

- A track matched to the energy deposit in the CAL was found in events in which an electron was reconstructed within the acceptance of the tracking detectors. This was

\footnotetext{
${ }^{2}$ The ZEUS coordinate system is a right-handed Cartesian system, with the $Z$ axis pointing in the proton-beam direction, referred to as the "forward direction", and the $X$ axis pointing left towards the centre of HERA. The coordinate origin is at the nominal interaction point.
}

done by restricting the distance of closest approach between the track extrapolated to the CAL surface and the energy cluster position to within $10 \mathrm{~cm}$, and requiring an electron track momentum greater than $3 \mathrm{GeV}$.

- The vertex position along the beam axis was in the range that was given by the nominal vertex position plus or minus three times the width of the vertex distribution approximated by a Gaussian. Both the nominal vertex position and the width of the distribution varied between the different data-taking periods. Typical values were $\left|Z_{\mathrm{vtx}}\right|<30 \mathrm{~cm}$. This condition helped to select events consistent with $e p$ interactions.

- $P_{T, \text { miss }} / \sqrt{E_{T}}<2.5 \mathrm{GeV}^{1 / 2}$, where $P_{T \text {, miss }}$ is the missing transverse momentum as measured with the CAL and $E_{T}$ is the total transverse energy in the CAL. This cut removed cosmic-ray events and beam-related background.

- $38<\left(E-P_{Z}\right)<65 \mathrm{GeV}$, where $E$ is the total energy, $E=\sum_{i} E_{i}$, and $P_{Z}$ is the $Z$-component of the vector $\boldsymbol{P}=\sum_{i} \boldsymbol{p}_{i}$. The sums run over all clusters of energy deposits in the CAL. This cut removed events with large initial-state radiation and further reduced the background from photoproduction.

In addition, events were rejected if a second electron candidate with azimuthal separation $\Delta \phi>3$ from the first candidate was found, a ratio of transverse momenta of the two candidates between 0.8 and 1.2 was measured, and, in addition, the rest of the CAL energy, besides the two electromagnetic energy clusters, was below $3 \mathrm{GeV}$. This condition removed elastic Compton scattering events $(e p \rightarrow e \gamma p)$.

\subsection{Jet selection}

The $k_{T}$ cluster algorithm [45] was used in the longitudinally invariant inclusive mode [46] to reconstruct jets in the hadronic final state assuming massless objects. In data, the algorithm was applied to the CAL cells after excluding those associated with the scattered-electron candidate. The jet search was performed in the $\eta-\varphi$ plane of the Breit frame. The jet variables were defined according to the Snowmass convention [47].

After reconstructing the jet variables in the Breit frame, the massless four-momenta were boosted into the laboratory frame, where the transverse energy, $E_{T, \mathrm{LAB}}^{\mathrm{jet}}$, and the pseudorapidity, $\eta_{\mathrm{LAB}}^{\text {jet }}$, of each jet were calculated. Energy corrections $[3,48,49]$ were then applied to the jets in the laboratory frame and propagated into $E_{T, \mathrm{~B}}^{\mathrm{jet}}$, the transverse jet energy in the Breit frame, in order to compensate for energy losses in the inactive material in front of the CAL.

The following cuts were applied to select a clean sample of high- $Q^{2}$ DIS jet events:

- Events were removed from the sample if the distance $\Delta R$ of any of the jets to the electron candidate in the 
$\eta-\varphi$ plane of the laboratory frame was smaller than 1 unit, $\Delta R=\sqrt{\left(\eta_{\mathrm{LAB}}^{\text {jet }}-\eta^{e}\right)^{2}+\left(\varphi_{\mathrm{LAB}}^{\mathrm{jet}}-\varphi^{e}\right)^{2}}<1$, where $\varphi^{e}$ and $\eta^{e}$ are the azimuthal angle and pseudorapidity of the scattered-electron candidate, respectively. This requirement removed some background from photoproduction and improved the purity of the sample.

- Events were removed from the sample if a jet was in the backward region of the detector $\left(\eta_{\mathrm{LAB}}^{\mathrm{jet}}<-1\right)$. This requirement removed events in which a radiated photon from the electron was misidentified as a jet in the Breit frame.

- $E_{T, \mathrm{LAB}}^{\mathrm{jet}}>3 \mathrm{GeV}$. This cut removed a small number of jets for which the uncertainty on the energy correction was large.

The dijet sample was then selected requiring the events to fulfil the following conditions (which also define the dijet phase space of the measurement):

- At least two jets in the pseudorapidity range $-1<$ $\eta_{\mathrm{LAB}}^{\mathrm{jet}}<2.5$ were found.

- Of these jets, the two with the highest transverse energies in the Breit frame were required to have $E_{T, \mathrm{~B}}^{\mathrm{jet}}>8 \mathrm{GeV}$.

- The invariant dijet mass, $M_{j j}$, of the two highest-transverse-energy jets in the event was required to exceed $20 \mathrm{GeV}$. This requirement was introduced to make the theoretical calculations infrared insensitive. Despite this cut the NLO QCD calculations in the region $0.65<\eta^{*}<2$ still exhibited infrared sensitivity (Sect. 5).

The final sample consisted of 31440 dijet events.

\section{Monte Carlo simulations and data corrections}

Samples of Monte Carlo (MC) events were generated to determine the response of the detector to jets of hadrons and the correction factors necessary to obtain the hadron-level jet cross sections. The hadron level is defined in terms of hadrons with lifetime $\tau \geq 10 \mathrm{ps}$. The generated events were passed through the GEANT-based [50] ZEUS detector- and trigger-simulation programs [21]. They were reconstructed and analysed by the same program chain as the data.

Neutral current DIS events including radiative effects were simulated using the HERACLES 4.6.1 [51, 52] program with the DJANGOH $1.1[53,54]$ interface to the hadronisation programs. HERACLES includes corrections for initial- and final-state radiation, vertex and propagator terms, and two-boson exchange. The QCD cascade is simulated using the colour-dipole model (CDM) [55-58] including the LO QCD diagrams as implemented in ARIADNE 4.08 [59, 60] and, alternatively, with the matrix-element plus parton-shower (MEPS) approach of LEPTO 6.5 [61]. The CTEQ5D [62] proton PDFs were used for these simulations. Fragmentation into hadrons is performed using the Lund string model [63] as implemented in JETSET 7.4 [64-67].

The jet search was performed on the MC events using the energy measured in the CAL cells in the same way as for the data. The same jet algorithm was also applied to the final-state particles (hadron level) and to the partons available after the parton shower simulation (parton level).

The data were corrected to the hadron level and for QEDradiative effects using bin-by-bin correction factors obtained from the MC samples. For this approach to be valid, the uncorrected distributions of the data must be well described by the MC simulations. This condition was in general satisfied by both the ARIADNE and LEPTO MC. Figures 1, 2, and 3 show comparisons of data with MC simulations for all observables in which cross sections are presented in this paper. The MC simulations give a good description of all the data distributions. The LEPTO model gives a slightly better description of the data and was thus used as the default model; ARIADNE was then used to estimate the systematic effect on the correction procedure due to the parton-shower model. In all cases, the correction factors differed from unity by $(5-30) \%$. These correction factors took into account the efficiency of the trigger, the selection criteria and the purity
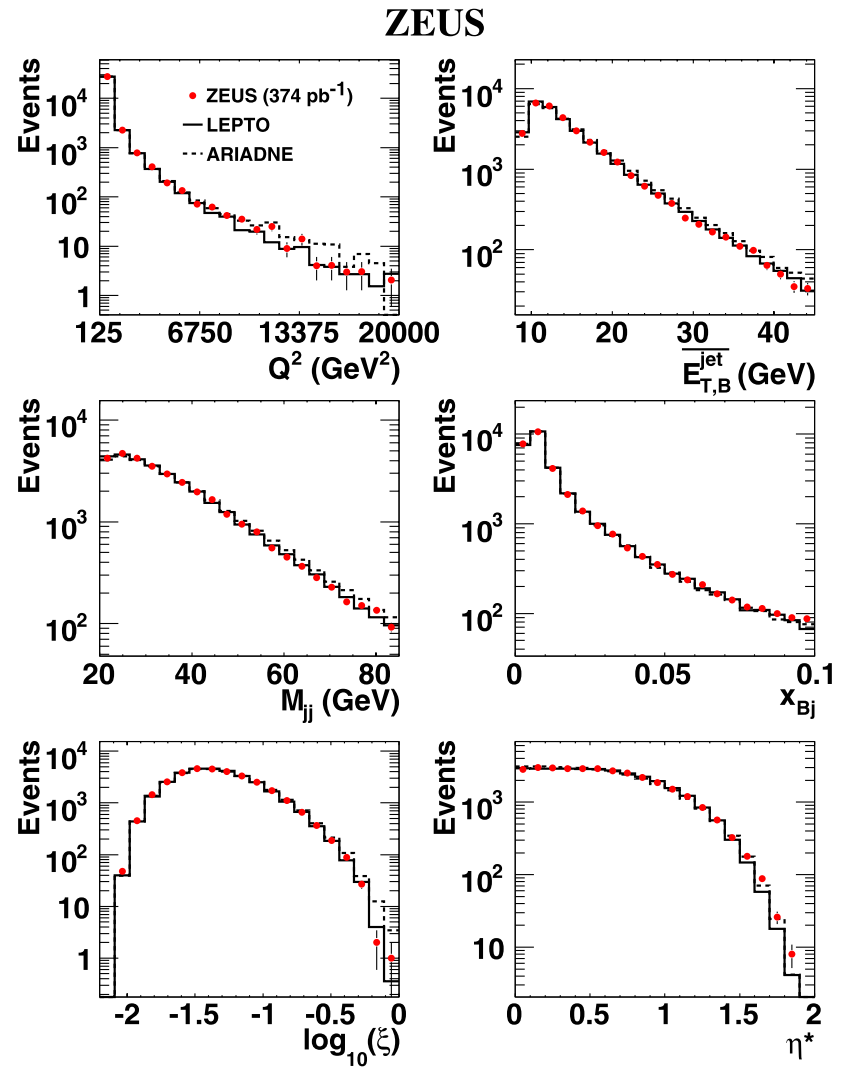

Fig. 1 Uncorrected data distributions for inclusive dijet production (dots). For comparison, the predictions of the ARIADNE (dashed histograms) and LEPTO (solid histograms) MC models are also included 


\section{ZEUS}
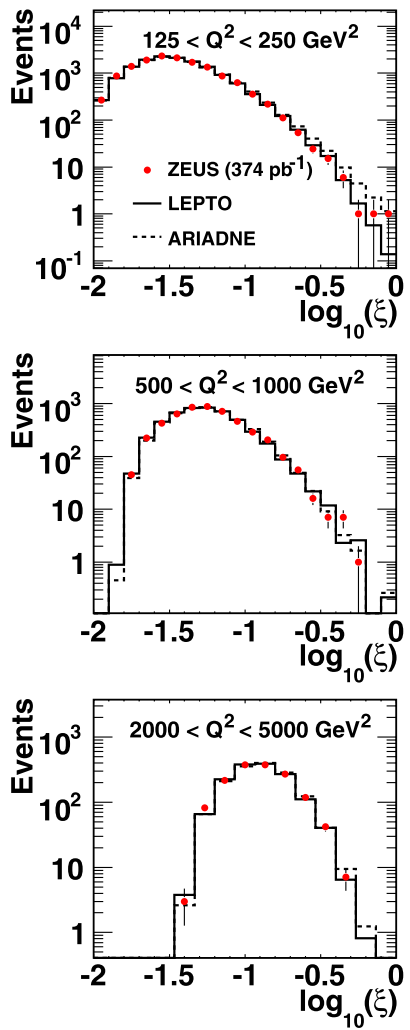
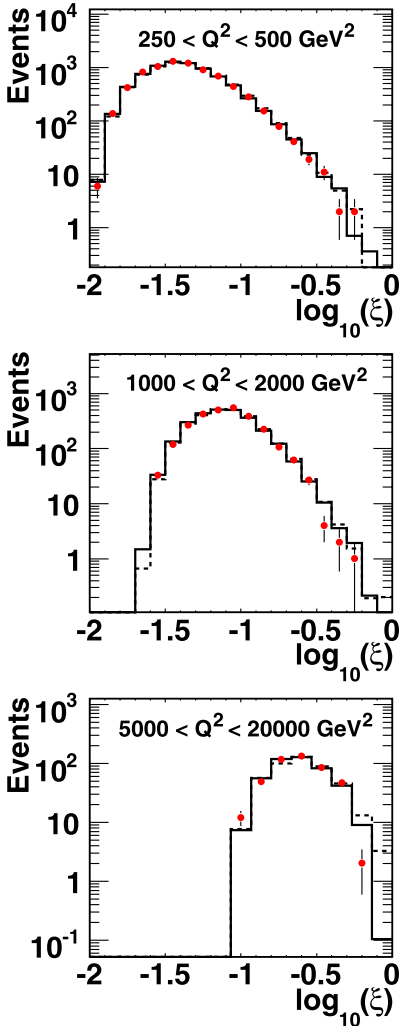

Fig. 2 Comparison of uncorrected data (dots) and MC model predictions for distributions of $\log _{10}(\xi)$ in different regions of $Q^{2}$. For comparison, the predictions of the ARIADNE (dashed histograms) and LEPTO (solid histograms) MC models are also included

and efficiency of the jet reconstruction. The QED corrections typically amounted to between 3 and $6 \%$.

Starting in 2004, HERA provided longitudinally polarised lepton beams. The effect of the polarisation on the measured data events was corrected for by adjusting the data event weights with the ratio of the predictions for the unpolarised and polarised cross sections as determined with the HECTOR program [68].

\section{NLO QCD calculations}

Next-to-leading-order $\left(\mathcal{O}\left(\alpha_{s}^{2}\right)\right)$ QCD calculations were obtained using the program NLOJET++ [69]. The calculations were performed in the $\overline{\mathrm{MS}}$ renormalisation and factorisation schemes. The number of flavours was set to five and the factorisation scale was chosen to be $\mu_{F}=Q$. Calculations with different choices of the renormalisation scale, $\mu_{R}$, were performed: the default choice was $\mu_{R}^{2}=Q^{2}+{\overline{E_{T, \mathrm{~B}}^{\mathrm{jet}}}}^{2}$. Alternatively, the scales $Q^{2}$ and ${\overline{E_{T, \mathrm{~B}}^{\mathrm{jet}}}}^{2}$ were investigated. The strong coupling constant was calculated at two loops with $\Lambda_{\frac{(5)}{\mathrm{MS}}}=226 \mathrm{MeV}$, corresponding to $\alpha_{S}\left(M_{Z}\right)=0.118$.

\section{ZEUS}
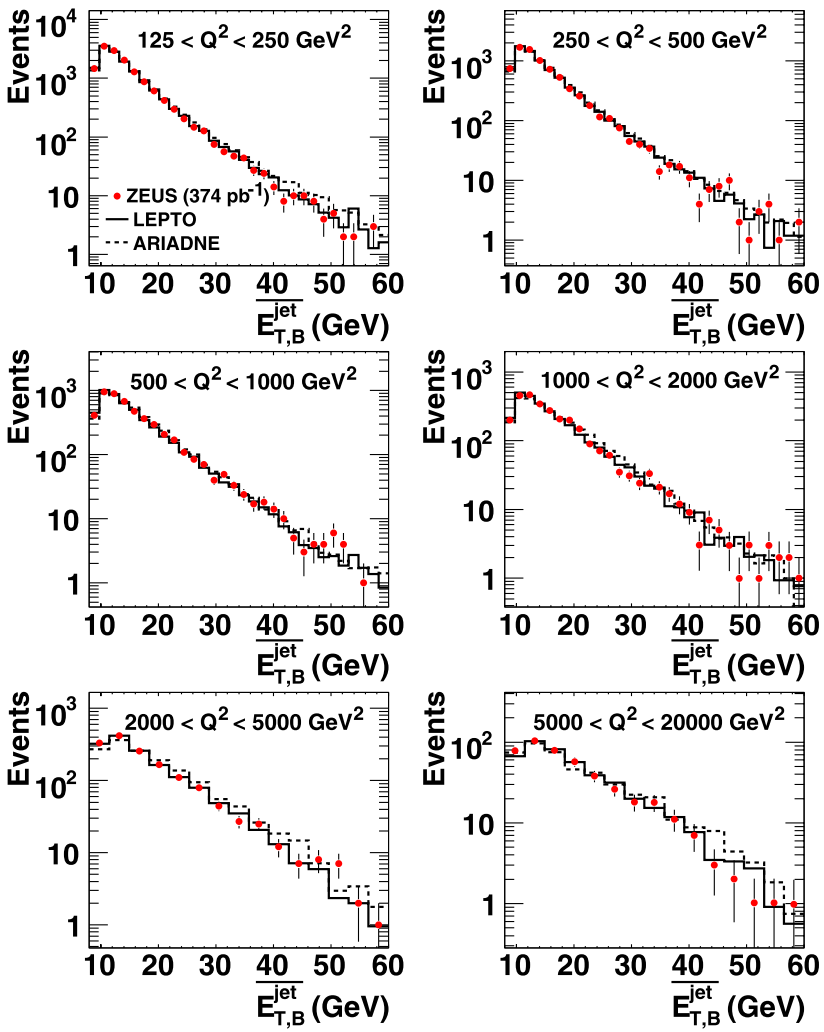

Fig. 3 Comparison of uncorrected data (dots) and MC model predictions for distributions of $\overline{E_{T, \mathrm{~B}}^{\text {jet }}}$ in different regions of $Q^{2}$. For comparison, the predictions of the ARIADNE (dashed histograms) and LEPTO (solid histograms) MC models are also included

The calculations were performed using the CTEQ6.6 [70] parameterisations of the proton PDFs. The $k_{T}$ cluster algorithm was also applied to the partons in the events generated by NLOJET++ in order to obtain the jet cross-section predictions. The predictions of NLOJET++ were cross-checked with the DISENT program [71]. Both programs agreed to better than $1 \%$.

The lack of sensitivity to the infrared cutoff of the NLO QCD calculations was verified by determining the total inclusive dijet cross section as a function of the $M_{\mathrm{jj}}$ cut in each analysis bin separately. Except for the two highest $\eta^{*}$ bins, the theoretical predictions were found to be infrared insensitive [72].

The data presented in this paper are, among others, intended for the use in QCD PDF fits, aiming specifically at a further improvement of the uncertainty on the gluon density at large values of $x$. In order to demonstrate the usefulness of the data for this purpose, Figs. 4 and 5 show, as a function of the variables $\xi$ and $\overline{E_{T, \mathrm{~B}}^{\mathrm{jet}}}$ in different regions of $Q^{2}$, the NLO predictions for the fraction of events which are initiated by a gluon from the proton using the CTEQ6.6 PDFs. This gluon fraction ranges from about $75 \%$ at $125<Q^{2}<250 \mathrm{GeV}^{2}$ 


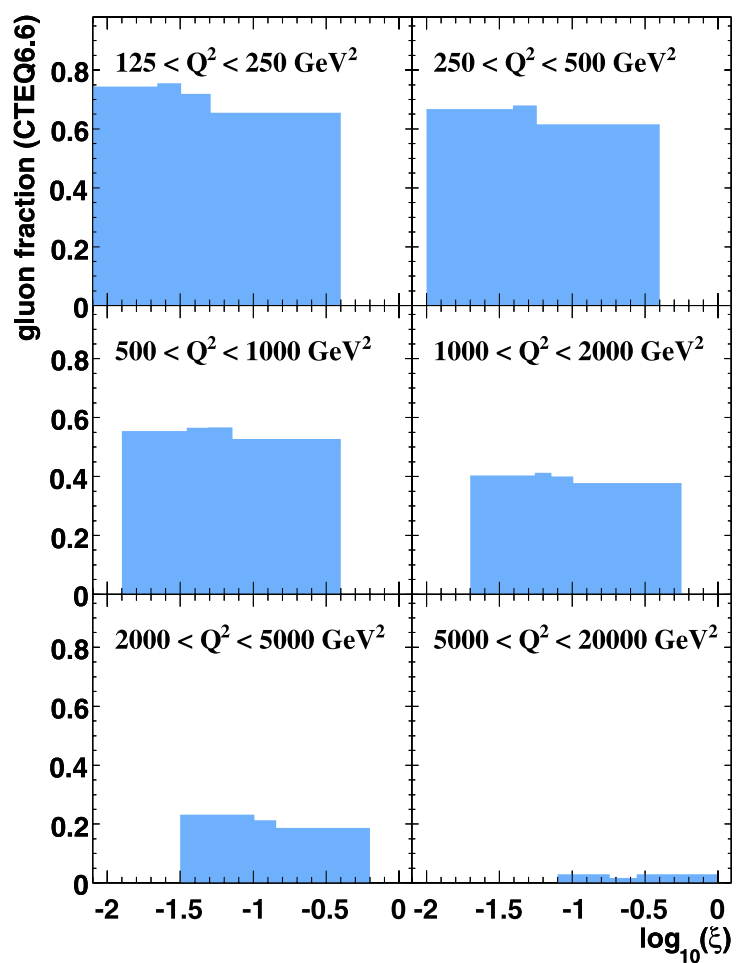

Fig. 4 The fraction of gluon-induced events as a function of $\log _{10}(\xi)$ as predicted by the CTEQ6.6 PDFs in different regions of $Q^{2}$

and small $\xi$ to about $5 \%$ at the highest $Q^{2}$ above $5000 \mathrm{GeV}^{2}$, where $\xi$ is approximately confined to values above 0.1 . In the lower $Q^{2}$ regions, the gluon fraction is also significant for large values of $\xi$. Since this region is not statistically limited, precise input for the PDF fits can be expected. Figure 6 shows the relative CTEQ6.6 PDF uncertainty, the uncertainty due to missing higher orders in the calculation estimated by variation of $\mu_{R}$ and the theoretical predictions from MSTW2008 [73], ZEUS-JETS [20] and ZEUS-S [74]. The corresponding uncertainties for the latter three PDF sets are not shown. The CTEQ6.6 PDF uncertainty and the observed spread between the various PDF sets is in some regions of the considered dijet phase space larger than the uncertainty arising from missing higher orders.

The measurements refer to jets of hadrons, whereas the NLO QCD calculations refer to jets of partons. The QCD predictions were corrected to the hadron level using the $\mathrm{MC}$ hadronisation model described in the previous section to give multiplicative factors, $C_{\text {hadr }}$, defined as the ratio of the cross section for jets of hadrons over that for jets of partons. The ratios obtained with ARIADNE and LEPTO were averaged to obtain the $C_{\text {hadr }}$ factors, which differ from unity by less than $5 \%$.

Neither NLOJET++ nor Disent includes the contributions from $Z^{0}$ exchange; MC simulated events with and without $Z^{0}$ exchange were used to include this effect in

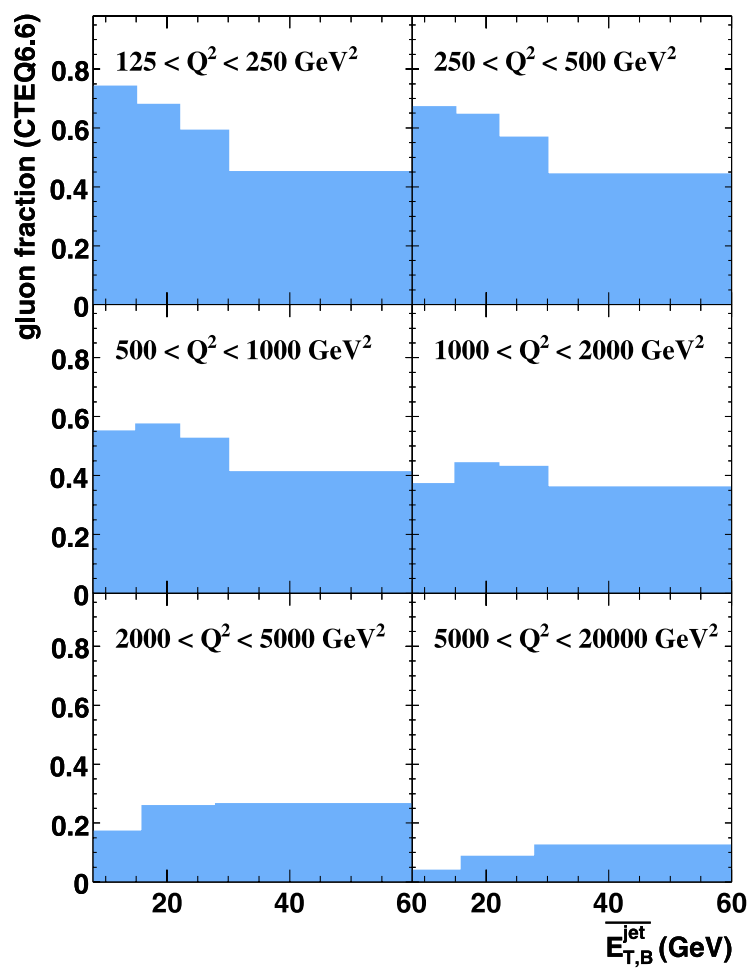

Fig. 5 The fraction of gluon-induced events as a function of $\overline{E_{T, \mathrm{~B}}^{\text {jet }}}$ as predicted by the CTEQ6.6 PDFs in different regions of $Q^{2}$

the $\mathrm{pQCD}$ predictions. In the following, $\mathrm{pQCD}$ calculations refer to the fully corrected predictions, unless otherwise stated.

Several sources of uncertainty in the theoretical predictions were considered:

- The uncertainty on the NLO QCD calculations due to terms beyond NLO, estimated by varying $\mu_{R}$ by a factor of two up and down, was below $\pm 6 \%$ at low $Q^{2}$ and low $E_{T, \mathrm{~B}}^{\mathrm{jet}}$ and decreased to below $\pm 3 \%$ in the highest- $Q^{2}$ region.

- The uncertainty on the NLO QCD calculations due to that on $\alpha_{s}\left(M_{Z}\right)$ was estimated by repeating the calculations using two additional sets of proton PDFs, CTEQ6.6A3 and CTEQ6.6A2, determined assuming $\alpha_{s}\left(M_{Z}\right)=0.114$ and 0.122 , respectively. The difference between the calculations using these sets and CTEQ6.6 was scaled to reflect the current uncertainty on $\alpha_{s}$ [75]. The resulting uncertainty on the cross sections was mostly below $\pm 3 \%$.

- The uncertainty on the modelling of the parton shower was estimated as half the difference between the correction factors calculated from the LEPTO and ARIADNE models. The resulting uncertainty on the cross sections was typically less than $2 \%$.

- The uncertainty on the NLO calculations due to the proton PDFs was estimated by repeating the calcula- 


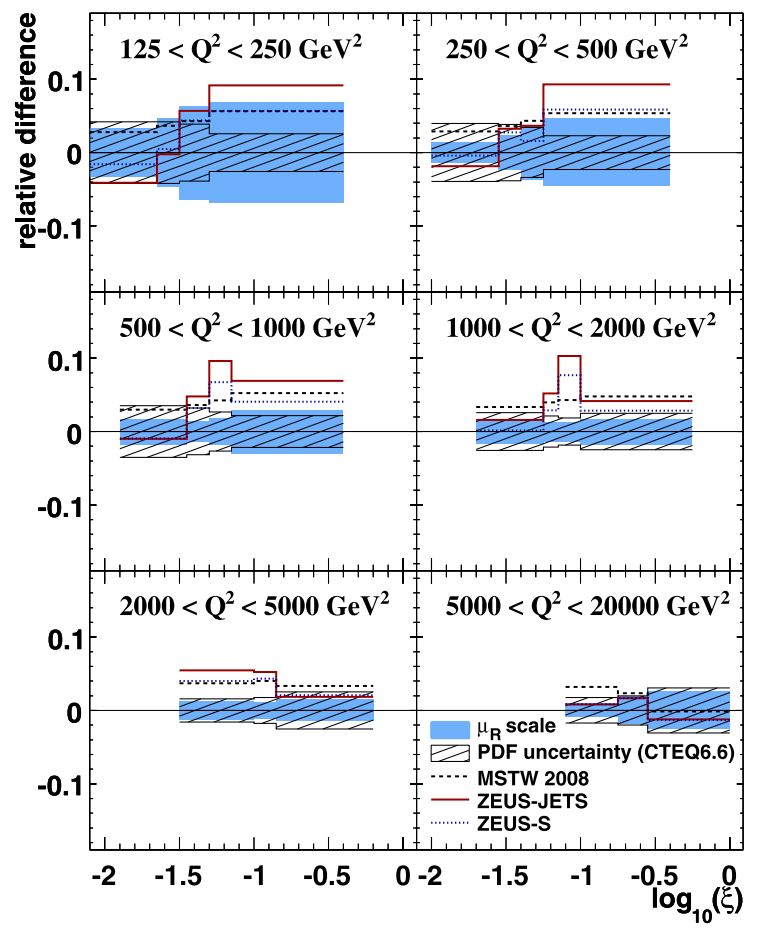

Fig. 6 The relative CTEQ6.6 PDF uncertainty, the relative uncertainty due to missing higher orders estimated by a variation of $\mu_{R}$ and the theoretical predictions from different PDF sets relative to those obtained with CTEQ6.6 as functions of $\log _{10}(\xi)$ in different regions of $Q^{2}$

tions using 44 additional sets from the CTEQ6.6 analysis, which takes into account the statistical and correlated systematic experimental uncertainties of each data set used in the determination of the proton PDFs. The resulting uncertainty on the cross sections was about $\pm 4 \%$ at low $Q^{2}$ and decreased to around $\pm 2 \%$ at high $Q^{2}$.

- The uncertainty of the calculations in the value of $\mu_{F}$ was estimated by repeating the calculations with $\mu_{F}=$ $Q / 2$ and $2 Q$. The effect on the calculations was negligible.

The total theoretical uncertainty was obtained by adding in quadrature the individual uncertainties listed above.

\section{Experimental uncertainties}

The following sources of systematic uncertainty were considered for the measured cross sections [72]:

- The uncertainty on the absolute energy scale of the jets was estimated to be $\pm 1 \%$ for $E_{T, \mathrm{LAB}}^{\mathrm{jet}}>10 \mathrm{GeV}$ and $\pm 3 \%$ for lower $E_{T, \mathrm{LAB}}^{\mathrm{jet}}$ values $[4,49,72,76]$. The resulting uncertainty on the cross sections was about $\pm 4 \%$ and increased to approximately $\pm 6 \%$ in certain regions of the dijet phase space.
- The uncertainty in the absolute energy scale of the electron candidate was estimated to be $\pm 1 \%$ [77] $( \pm 2 \%$ [78]) for the data from the years 1998-2000 (2004-2007). The resulting uncertainty was below $\pm 1 \%$.

- The differences in the results obtained by using either ARIADNE or LEPTO to correct the data for detector effects were typically below $\pm 5 \%$.

- The analysis was repeated using an alternative technique [79] to select the scattered-electron candidate. The resulting uncertainty was typically below $\pm 1 \%$.

- The $E_{T, \mathrm{LAB}}^{\mathrm{jet}}$ cut was changed to 2 and $4 \mathrm{GeV}$. The resulting uncertainty was mostly smaller than $\pm 1 \%$.

- The uncertainty due to the selection cuts was estimated by varying the values of the cuts within the resolution of each variable. The effect on the cross sections was in general below $\pm 2 \%$.

- The combined, luminosity-weighted systematic error on the polarisation measurement was $3.9 \%$. The effect on the cross sections was negligible.

- The simulation of the first-level trigger was corrected in order to match the measured efficiency in the data. The systematic effect on the cross sections was typically less than $1 \%$.

The systematic uncertainties not associated with the absolute energy scale of the jets were added in quadrature. Figure 7 shows the statistical uncertainty, the correlated systematic uncertainty which is caused by the jet energy scale and the quadratic sum of the correlated and uncorrelated systematic uncertainties as a function of $Q^{2}$. Except for the high- $Q^{2}$ region, the correlated uncertainty was the dominating contribution to the total experimental uncertainty. In addition, there was an overall normalisation uncertainty of $\pm 2.2 \%$ for the $1998-2000$ data and of $\pm 2.6 \%$ for the 2004 2007 data. Therefore, the combined, luminosity-weighted average systematic uncertainty on the luminosity measurement was $\pm 2.5 \%$, which was not included in the crosssection figures or the tables.

\section{Results}

The differential inclusive dijet cross sections were measured in the kinematic region $125<Q^{2}<20000 \mathrm{GeV}^{2}$ and $0.2<y<0.6$. The jets were reconstructed using the $k_{T}$ cluster algorithm in the longitudinally invariant inclusive mode and the cross sections refer to jets with $E_{T, \mathrm{~B}}^{\mathrm{jet}}>8 \mathrm{GeV}$ and $-1<\eta_{\mathrm{LAB}}^{\text {jet }}<2.5$. The invariant dijet mass of the two highest-transverse-energy jets in the event was required to be greater than $20 \mathrm{GeV}$. These cross sections were corrected for detector and QED radiative effects and the running of $\alpha_{\mathrm{em}}$. 
Fig. 7 The statistical uncertainty, $\delta_{\text {stat }}$, the correlated uncertainty associated with the energy scale of the jets, $\delta_{\mathrm{ES}}$, and the quadratic sum of the correlated and uncorrelated, $\delta_{\text {syst }}$, systematic uncertainties, $\sqrt{\delta_{\mathrm{ES}}^{2}+\delta_{\text {syst }}^{2}}$, as functions of $Q^{2}$

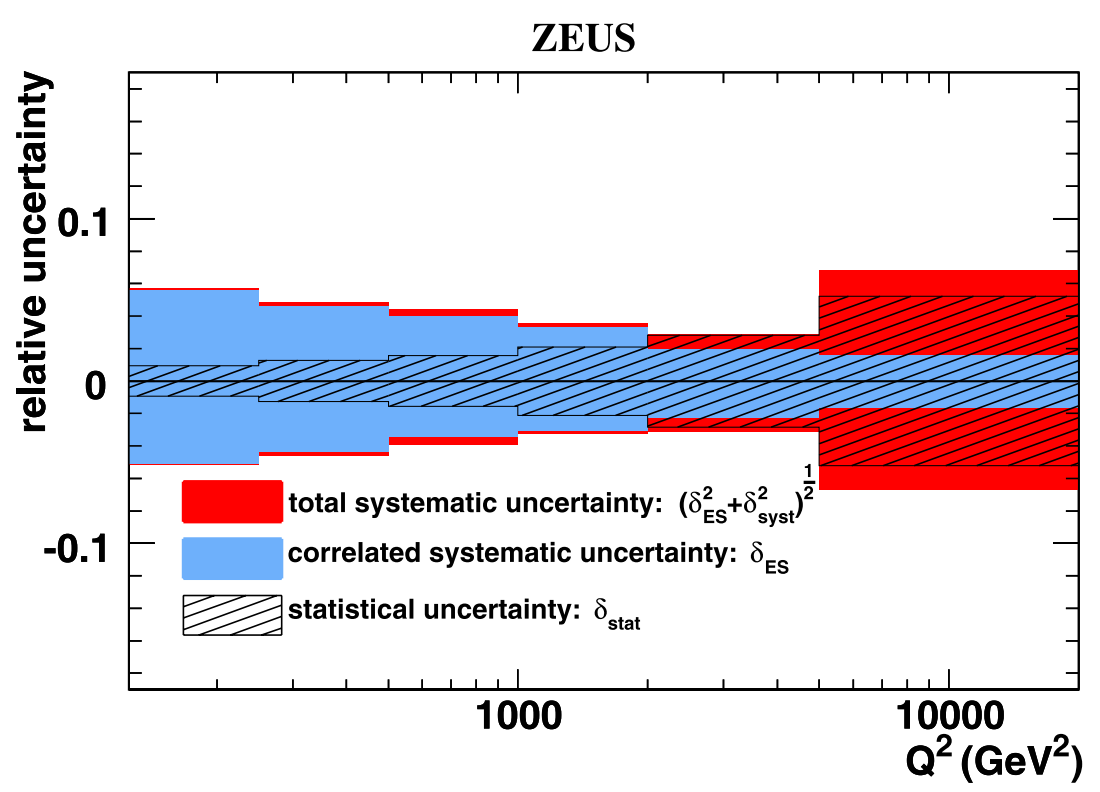

\subsection{Single-differential dijet cross sections}

The measurements of the single-differential inclusive dijet cross sections are presented in Figs. 8 to 10 and Tables 1 , $2,3,4,5$, and 6 as functions of several kinematic and dijet variables. Single-differential cross sections are shown for $Q^{2}, x_{\mathrm{Bj}}$, the mean transverse jet energy in the Breit frame of the two jets, $\overline{E_{T, \mathrm{~B}}^{\mathrm{jet}}}$, the dijet invariant mass, $M_{\mathrm{jj}}$, the halfdifference of the jet pseudorapidities in the Breit frame, $\eta^{*}$, and the logarithm of the variable $\xi$. The data are compared to NLO QCD calculations. The relative differences between the measured differential cross sections and the NLO QCD calculations are also shown.

The single-differential dijet cross-sections $d \sigma / d Q^{2}$ and $d \sigma / d x_{\mathrm{Bj}}$ are shown in Figs. 8a and b. The cross-section $d \sigma / d Q^{2}$ has total experimental systematic uncertainties of the order of $5 \%(7 \%)$ at low (high) values of $Q^{2}$. The total theoretical uncertainty is of the order of $7 \%(4 \%)$ at low (high) $Q^{2}$.

For the cross-section $d \sigma / d x_{\mathrm{Bj}}$, most of the data points have experimental uncertainties of less than $5 \%$, and also the precision of the theory predictions is better than $5 \%$ over most of the $x_{\mathrm{Bj}}$ range.

Figures $9 \mathrm{a}$ and $\mathrm{b}$ show the single-differential dijet crosssections $d \sigma / d \overline{E_{T, \mathrm{~B}}^{\mathrm{jet}}}$ and $d \sigma / d M_{\mathrm{jj}}$. These measurements are particularly well suited for testing the matrix elements in the perturbative calculations. Mean transverse jet energies $\overline{E_{T, \mathrm{~B}}^{\text {jet }}}$ (dijet invariant masses $M_{\mathrm{jj}}$ ) of up to $60 \mathrm{GeV}$ $(120 \mathrm{GeV})$ are reached with this measurement. At the largest values of $\overline{E_{T, \mathrm{~B}}^{\mathrm{jet}}}\left(M_{\mathrm{jj}}\right)$, experimental uncertainties of $8 \%$ $(5 \%)$ are achieved; for smaller values, the uncertainties are even smaller. The theoretical uncertainties are approx- imately constant over the range studied and are of the order of $(5-7) \%$.

The differential dijet cross-section as a function of $\eta^{*}$ is shown in Fig. 3a. The experimental uncertainties are always below $5 \%$, the total theoretical uncertainty is also typically around $5 \%$. The theoretical predictions for the last two $\eta^{*}$ bins were removed from the plot due to infrared sensitivity.

The cross-section $d \sigma / d \log _{10}(\xi)$ (Fig. 3b) has similar uncertainties as the distributions described before and shows a maximum around $\log _{10}(\xi)=-1.5$. At lower and higher values, the cross section reflects the suppression by the transverse energy requirements in the selection and the decreasing quark and gluon densities, respectively.

All the measured differential cross sections are well described by NLO QCD predictions.

\subsection{Double-differential dijet cross sections}

Figures 11, 12, 13, and 14 show the measurements of double-differential dijet cross sections as functions of $\overline{E_{T, \mathrm{~B}}^{\mathrm{jet}}}$ and $\log _{10}(\xi)$ in different $Q^{2}$ regions (see Tables 7 and 8 ). These cross sections will provide valuable input for the extraction of the proton PDFs.

The $\log _{10}(\xi)$ distributions in different $Q^{2}$ regions in Fig. 11 show the same behaviour as the integrated $\log _{10}(\xi)$ distribution in Fig. 10b, with a distinct maximum at values that increase with increasing $Q^{2}$. The data are very precise-even in the highest $Q^{2}$ bin from 5000 to $20000 \mathrm{GeV}^{2}$ the experimental uncertainties are between 10 and $15 \%$ and originate equally from the statistical and the systematical uncertainty. At lower $Q^{2}$ values, the experimental uncertainties become as small as (2-3)\%. Fig- 
ZEUS

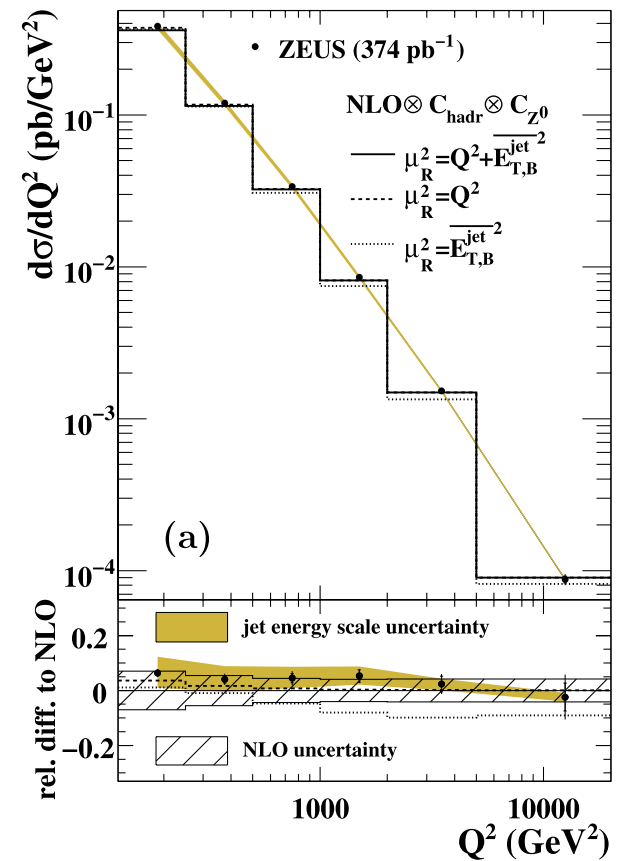

Fig. 8 The measured differential cross-sections a $d \sigma / d Q^{2}$ and b $d \sigma / d x_{\mathrm{Bj}}$ for inclusive dijet production with $E_{T, \mathrm{~B}}^{\mathrm{jet}}>8 \mathrm{GeV}$, $M_{\mathrm{jj}}>20 \mathrm{GeV}$ and $-1<\eta_{\mathrm{LAB}}^{\mathrm{jet}}<2.5$ (dots), in the kinematic range given by $0.2<y<0.6$ and $125<Q^{2}<20000 \mathrm{GeV}^{2}$. The inner error bars represent the statistical uncertainty. The outer error bars show the statistical and systematic uncertainties, not associated with the uncertainty on the absolute energy scale of the jets, added in quadrature. The shaded bands display the uncertainties due to the absolute energy

Table 1 The measured differential cross-sections $d \sigma / d Q^{2}$ for inclusive dijet production. The statistical, uncorrelated systematic and jetenergy-scale (ES) uncertainties are shown separately. The multiplicative corrections, $C_{\mathrm{QED}}$, which have been applied to the data and the

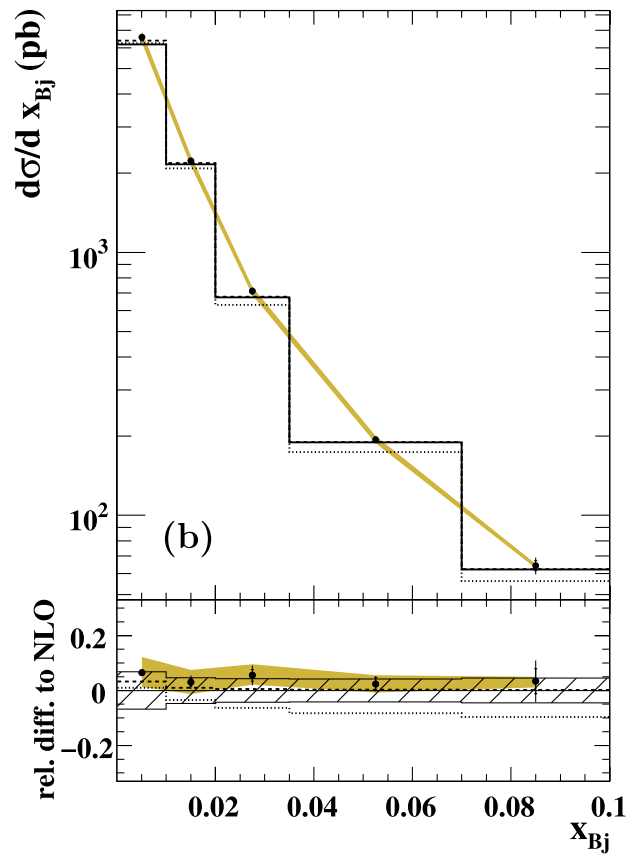

scale of the jets. The NLO QCD calculations with $\mu_{R}^{2}=Q^{2}+{\overline{E_{T, \mathrm{~B}}^{\mathrm{jet}}}}^{2}$ (solid lines), $\mu_{R}^{2}=Q^{2}$ (dashed lines) and $\mu_{R}^{2}={\overline{E_{T, \mathrm{~B}}^{\mathrm{jet}}}}^{2}$ (dotted lines), corrected for hadronisation effects and $Z^{0}$ exchange and using the CTEQ6.6 parameterisations of the proton PDFs, are also shown. The lower parts of the figures show the relative differences with respect to the NLO QCD calculations with $\mu_{R}^{2}=Q^{2}+{\overline{E_{T, \mathrm{~B}}^{\mathrm{jet}}}}^{2}$. The hatched bands display the total theoretical uncertainty

corrections for hadronisation and $Z^{0}$ effects to be applied to the partonlevel NLO QCD calculations, $C_{\mathrm{hadr}} \cdot C_{\mathrm{Z}^{0}}$, are shown in the last two columns

\begin{tabular}{|c|c|c|c|c|c|c|}
\hline \multirow{2}{*}{$\begin{array}{l}Q^{2} \text { bin } \\
\left(\mathrm{GeV}^{2}\right)\end{array}$} & \multicolumn{4}{|l|}{$d \sigma / d Q^{2}$} & \multirow[b]{2}{*}{$C_{\mathrm{QED}}$} & \multirow[b]{2}{*}{$C_{\text {hadr }} \cdot C_{Z^{0}}$} \\
\hline & $\left(\mathrm{pb} / \mathrm{GeV}^{2}\right)$ & $\delta_{\text {stat }}$ & $\delta_{\text {syst }}$ & $\delta_{\mathrm{ES}}$ & & \\
\hline $125-250$ & 0.3843 & \pm 0.0036 & $\begin{array}{l}+0.0039 \\
-0.0040\end{array}$ & $\begin{array}{l}+0.0215 \\
-0.0195\end{array}$ & 0.97 & 0.95 \\
\hline $250-500$ & 0.1193 & \pm 0.0015 & $\begin{array}{l}+0.0019 \\
-0.0018\end{array}$ & $\begin{array}{l}+0.0055 \\
-0.0052\end{array}$ & 0.95 & 0.96 \\
\hline $500-1000$ & 0.03372 & \pm 0.00053 & $\begin{array}{l}+0.00065 \\
-0.00065\end{array}$ & $\begin{array}{l}+0.00135 \\
-0.00115\end{array}$ & 0.94 & 0.96 \\
\hline 1000-2000 & 0.00855 & \pm 0.00018 & $\begin{array}{l}+0.00010 \\
-0.00010\end{array}$ & $\begin{array}{l}+0.00029 \\
-0.00026\end{array}$ & 0.93 & 0.98 \\
\hline $2000-5000$ & 0.001523 & \pm 0.000043 & $\begin{array}{l}+0.000033 \\
-0.000033\end{array}$ & $\begin{array}{l}+0.000030 \\
-0.000034\end{array}$ & 0.93 & 1.03 \\
\hline 5000-20000 & 0.0000875 & \pm 0.0000046 & $\begin{array}{l}+0.0000058 \\
-0.0000057\end{array}$ & $\begin{array}{l}+0.0000014 \\
-0.0000015\end{array}$ & 0.92 & 1.09 \\
\hline
\end{tabular}

ure 12 shows the level of agreement between data and predictions: the theoretical uncertainties are typically between 5 and $10 \%$ and, within the combined uncertainties, the data are very well described by the theory.

The cross sections as functions of $\overline{E_{T, \mathrm{~B}}^{\mathrm{jet}}}$ in different regions of $Q^{2}$, shown in Fig. 13, fall over 2 to 3 orders of magnitude in the range considered, with a smaller slope for higher $Q^{2}$ values. The statistical precision of the data is between $2 \%$ at the lowest $\overline{E_{T, \mathrm{~B}}^{\mathrm{jet}}}$ and $Q^{2}$ and slightly above $10 \%$ at the highest values of these variables. The systematic uncertainties are mostly of the order of 3 to $5 \%$. The theoretical uncertainties (Fig. 14) are approximately constant in $\overline{E_{T, \mathrm{~B}}^{\mathrm{jet}}}$; 
Table 2 Inclusive dijet cross-sections $d \sigma / d x_{\mathrm{Bj}}$. Other details as in the caption to Table 1

Table 3 Inclusive dijet cross-sections $d \sigma / d \overline{E_{T, \mathrm{~B}}^{\mathrm{jet}}}$. Other details as in the caption to Table 1

Table 4 Inclusive dijet cross-sections $d \sigma / d M_{\mathrm{jj}}$. Other details as in the caption to Table 1

Table 5 Inclusive dijet cross-sections $d \sigma / d \eta^{*}$. Other details as in the caption to Table 1

\begin{tabular}{|c|c|c|c|c|c|c|}
\hline \multirow[t]{2}{*}{$x_{\mathrm{Bj}}$ bin } & \multicolumn{4}{|c|}{$d \sigma / d x_{\mathrm{Bj}}$} & \multirow[b]{2}{*}{$C_{\mathrm{QED}}$} & \multirow[b]{2}{*}{$C_{\text {hadr }} \cdot C_{Z^{0}}$} \\
\hline & $(\mathrm{pb})$ & $\delta_{\text {stat }}$ & $\delta_{\text {syst }}$ & $\delta_{\mathrm{ES}}$ & & \\
\hline $0.0001-0.01$ & 6580 & \pm 54 & $\begin{array}{l}+44 \\
-45\end{array}$ & $\begin{array}{l}+351 \\
-317\end{array}$ & 0.96 & 0.95 \\
\hline $0.01-0.02$ & 2229 & \pm 31 & $\begin{array}{l}+44 \\
-44\end{array}$ & $\begin{array}{l}+98 \\
-94\end{array}$ & 0.94 & 0.95 \\
\hline $0.02-0.035$ & 711 & \pm 14 & $\begin{array}{l}+19 \\
-20\end{array}$ & $\begin{array}{l}+27 \\
-23\end{array}$ & 0.94 & 0.96 \\
\hline $0.035-0.07$ & 193.8 & \pm 4.6 & $\begin{array}{l}+2.8 \\
-2.5\end{array}$ & $\begin{array}{l}+6.1 \\
-5.7\end{array}$ & 0.93 & 0.99 \\
\hline $0.07-0.1$ & 64.4 & \pm 2.8 & $\begin{array}{l}+3.8 \\
-3.8\end{array}$ & $\begin{array}{l}+1.0 \\
-1.4\end{array}$ & 0.92 & 1.03 \\
\hline
\end{tabular}

\begin{tabular}{lclllll}
\hline $\begin{array}{l}E_{T, \mathrm{~B}}^{\text {jet }} \\
(\mathrm{GeV})\end{array}$ & $\begin{array}{l}d \sigma / d \overline{E_{T, \mathrm{~B}}^{\text {jet }}} \\
(\mathrm{pb} / \mathrm{GeV})\end{array}$ & $\delta_{\text {stat }}$ & $\delta_{\text {syst }}$ & $\delta_{\mathrm{ES}}$ & $C_{\mathrm{QED}}$ & $C_{\text {hadr }} \cdot C_{\mathrm{Z}^{0}}$ \\
\hline $8-15$ & 10.650 & \pm 0.083 & $+_{-0.174}^{+0.174}$ & ${ }_{-0.495}^{+0.549}$ & 0.95 & 0.95 \\
$15-22$ & 3.595 & \pm 0.046 & ${ }_{-0.062}^{+0.060}$ & ${ }_{-0.134}^{+0.142}$ & 0.95 & 0.98 \\
$22-30$ & 0.848 & \pm 0.020 & ${ }_{-0.010}^{+0.011}$ & ${ }_{-0.026}^{+0.025}$ & 0.95 & 0.99 \\
$30-60$ & 0.0896 & \pm 0.0031 & ${ }_{-0.0027}^{+0.0027}$ & ${ }_{-0.0038}^{+0.0041}$ & 0.95 & 0.99 \\
\hline
\end{tabular}

\begin{tabular}{|c|c|c|c|c|c|c|}
\hline \multirow{2}{*}{$\begin{array}{l}M_{\mathrm{jj}} \text { bin } \\
(\mathrm{GeV})\end{array}$} & \multicolumn{4}{|l|}{$d \sigma / d M_{\mathrm{jj}}$} & \multirow[b]{2}{*}{$C_{\mathrm{QED}}$} & \multirow[b]{2}{*}{$C_{\mathrm{hadr}} \cdot C_{\mathrm{Z}}$} \\
\hline & $(\mathrm{pb} / \mathrm{GeV})$ & $\delta_{\text {stat }}$ & $\delta_{\text {syst }}$ & $\delta_{\mathrm{ES}}$ & & \\
\hline 20-30 & 5.048 & \pm 0.049 & $\begin{array}{l}+0.079 \\
-0.079\end{array}$ & $\begin{array}{l}+0.236 \\
-0.212\end{array}$ & 0.95 & 0.95 \\
\hline $30-45$ & 2.693 & \pm 0.028 & $\begin{array}{l}+0.038 \\
-0.038\end{array}$ & $\begin{array}{l}+0.130 \\
-0.121\end{array}$ & 0.95 & 0.97 \\
\hline $45-65$ & 0.726 & \pm 0.012 & $\begin{array}{l}+0.009 \\
-0.010\end{array}$ & $\begin{array}{l}+0.031 \\
-0.029\end{array}$ & 0.95 & 0.98 \\
\hline $65-120$ & 0.0681 & \pm 0.0020 & $\begin{array}{l}+0.0005 \\
-0.0005 \\
\end{array}$ & $\begin{array}{l}+0.0032 \\
-0.0031 \\
\end{array}$ & 0.95 & 0.97 \\
\hline
\end{tabular}

\begin{tabular}{|c|c|c|c|c|c|c|}
\hline \multirow[t]{2}{*}{$\eta^{*}$ bin } & \multicolumn{4}{|l|}{$d \sigma / d \eta^{*}$} & \multirow[b]{2}{*}{$C_{\mathrm{QED}}$} & \multirow[b]{2}{*}{$C_{\text {hadr }} \cdot C_{\mathrm{Z}}$} \\
\hline & $\overline{(\mathrm{pb})}$ & $\delta_{\text {stat }}$ & $\delta_{\text {syst }}$ & $\delta_{\mathrm{ES}}$ & & \\
\hline $0-0.2$ & 106.1 & \pm 1.6 & $\begin{array}{l}+0.9 \\
-0.8\end{array}$ & $\begin{array}{l}+4.3 \\
-3.8\end{array}$ & 0.95 & 0.96 \\
\hline $0.2-0.4$ & 105.4 & \pm 1.6 & $\begin{array}{l}+0.9 \\
-0.9\end{array}$ & $\begin{array}{l}+4.3 \\
-4.1\end{array}$ & 0.95 & 0.96 \\
\hline $0.4-0.65$ & 101.0 & \pm 1.4 & $\begin{array}{l}+0.6 \\
{ }_{-0.7}\end{array}$ & $\begin{array}{l}+4.1 \\
-4.0\end{array}$ & 0.96 & 0.97 \\
\hline $0.65-0.95$ & 78.2 & \pm 1.1 & $\begin{array}{l}+0.3 \\
-0.4\end{array}$ & $\begin{array}{l}+4.0 \\
-3.3\end{array}$ & 0.95 & 0.98 \\
\hline $0.95-2$ & 17.14 & \pm 0.27 & $\begin{array}{l}+0.42 \\
+0.42\end{array}$ & $\begin{array}{l}+1.06 \\
-1.02\end{array}$ & 0.95 & 0.93 \\
\hline
\end{tabular}

Table 6 Inclusive dijet cross-sections $d \sigma / d \log _{10}(\xi)$. Other details as in the caption to Table 1

\begin{tabular}{|c|c|c|c|c|c|c|}
\hline \multirow[t]{2}{*}{$\log _{10}(\xi)$ bin } & \multicolumn{4}{|c|}{$d \sigma / d \log _{10}(\xi)$} & \multirow[b]{2}{*}{$C_{\mathrm{QED}}$} & \multirow[b]{2}{*}{$C_{\text {hadr }} \cdot C_{\mathrm{Z}^{0}}$} \\
\hline & $(\mathrm{pb})$ & $\delta_{\text {stat }}$ & $\delta_{\text {syst }}$ & $\delta_{\mathrm{ES}}$ & & \\
\hline$-2-(-1.6)$ & 62.63 & \pm 0.91 & $\begin{array}{l}+0.81 \\
-0.86\end{array}$ & $\begin{array}{l}+3.27 \\
-2.83\end{array}$ & 0.97 & 0.95 \\
\hline$-1.6-(-1.45)$ & 143.3 & \pm 2.1 & $\begin{array}{l}+2.5 \\
-2.5\end{array}$ & $\begin{array}{l}+7.1 \\
-6.6\end{array}$ & 0.96 & 0.95 \\
\hline$-1.45-(-1.3)$ & 143.0 & \pm 2.1 & $\begin{array}{l}+1.0 \\
-0.8\end{array}$ & $\begin{array}{l}+7.3 \\
-6.1\end{array}$ & 0.95 & 0.96 \\
\hline$-1.3-(-1.1)$ & 109.9 & \pm 1.5 & $\begin{array}{l}+2.9 \\
-3.0\end{array}$ & $\begin{array}{l}+4.9 \\
-4.9\end{array}$ & 0.95 & 0.96 \\
\hline$-1.1-0$ & 17.40 & \pm 0.24 & $\begin{array}{l}+0.11 \\
-0.13 \\
\end{array}$ & $\begin{array}{l}+0.65 \\
-0.63 \\
\end{array}$ & 0.94 & 0.98 \\
\hline
\end{tabular}


Fig. 9 The measured differential cross-sections $\mathbf{a} d \sigma / d \overline{E_{T, \mathrm{~B}}^{\mathrm{jet}}}$ and $\mathbf{b} d \sigma / d M_{\mathrm{jj}}$ for inclusive dijet production. Other details as in the caption to Fig. 8

Fig. 10 The measured differential cross-sections a $d \sigma / d \eta^{*}$ and $\mathbf{b} d \sigma / d \log _{10}(\xi)$ for inclusive dijet production. In the last $\eta^{*}$ bins the NLO QCD predictions are not plotted for reasons explained in the text. Other details as in the caption to Fig. 8

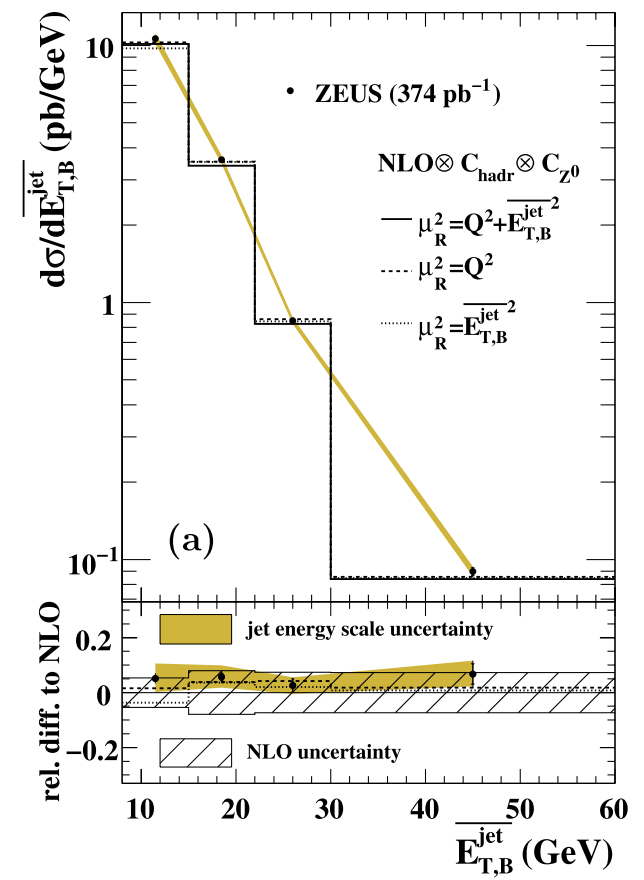

ZEUS

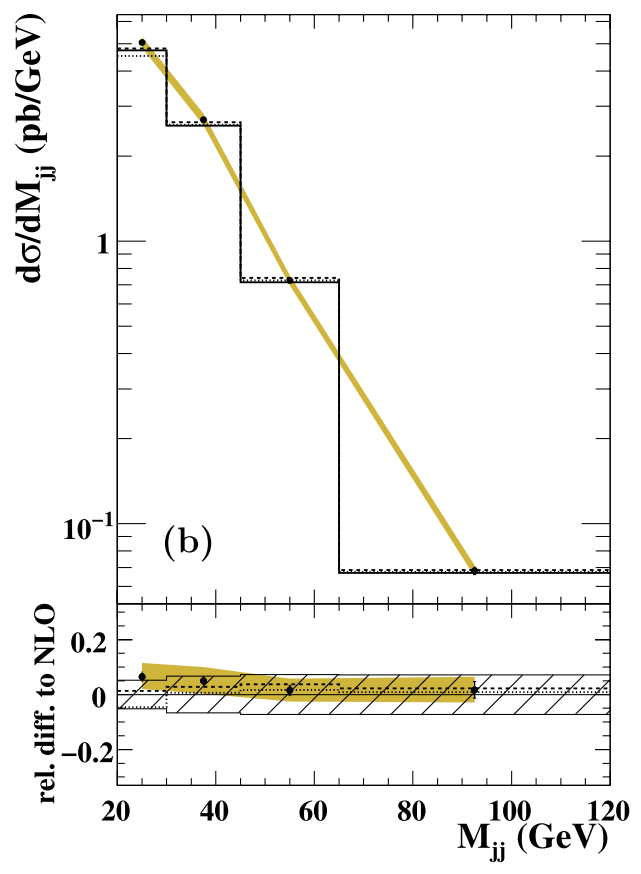

\section{ZEUS}

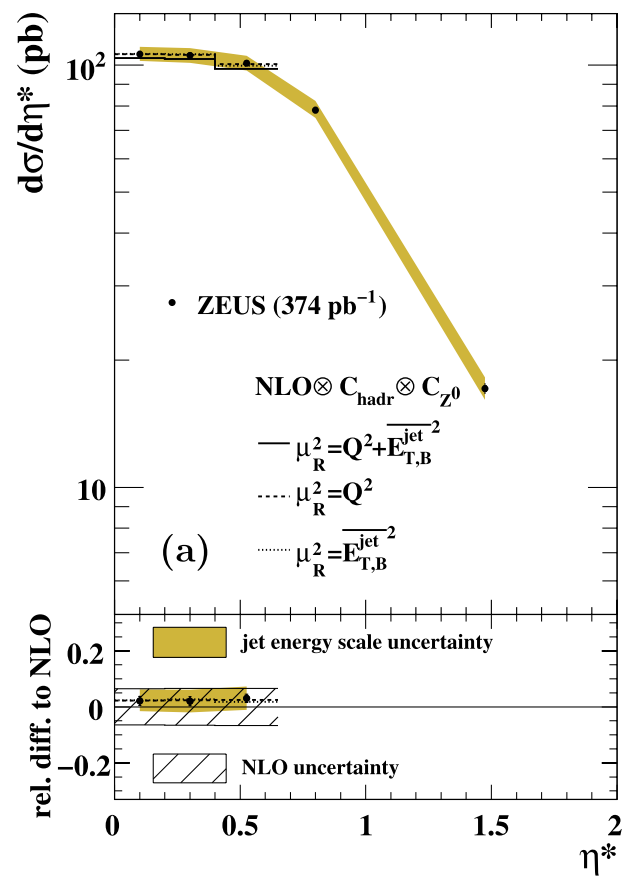

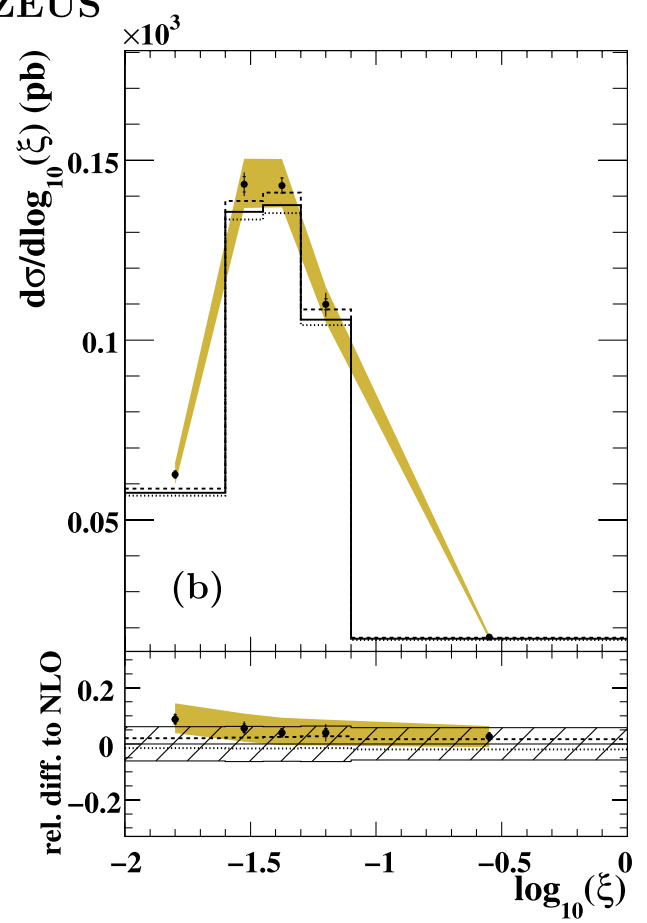


Fig. 11 The measured differential cross-section $d \sigma / d \log _{10}(\xi)$ for inclusive dijet production in different regions of $Q^{2}$. Other details as in the caption to Fig. 8
Fig. 12 Relative differences between the measured differential cross-sections $d \sigma / d \log _{10}(\xi)$ presented in Fig. 11 and the NLO QCD calculations with $\mu_{R}^{2}=Q^{2}+{\overline{E_{T, \mathrm{~B}}^{\mathrm{jet}}}}^{2}$. Other details as in the caption to Fig. 8

\section{ZEUS}

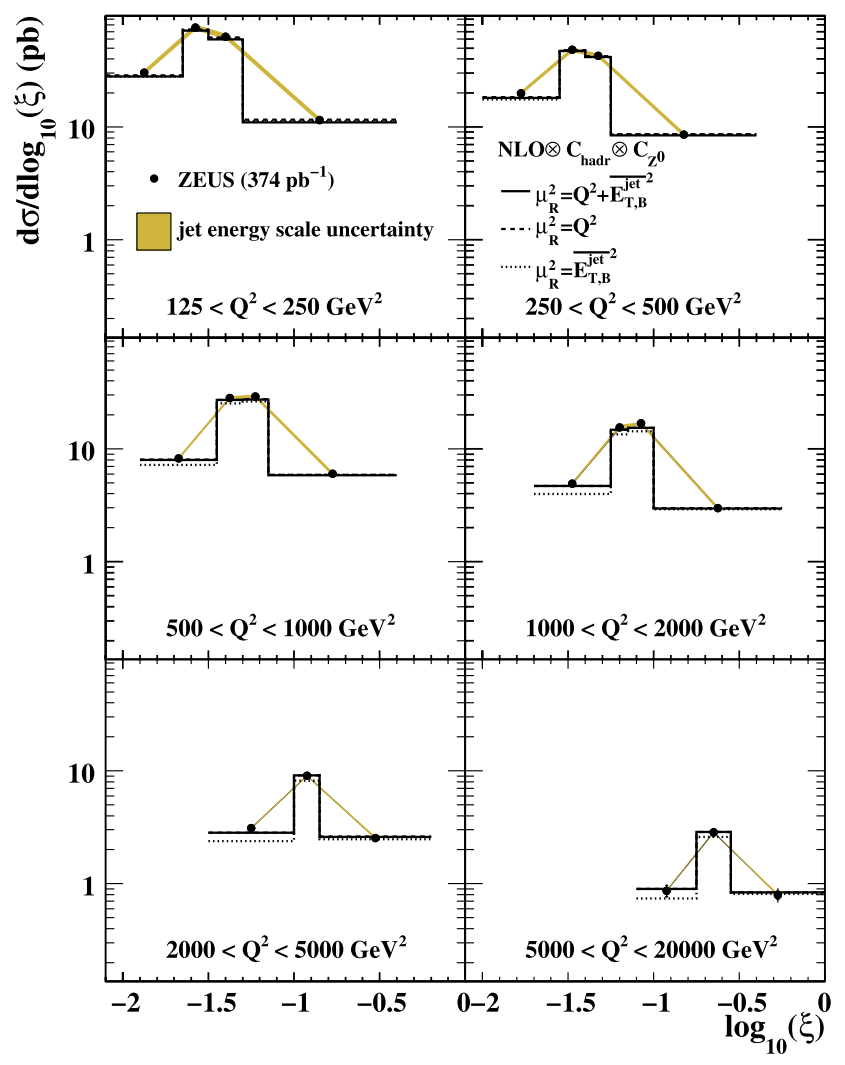

\section{ZEUS}

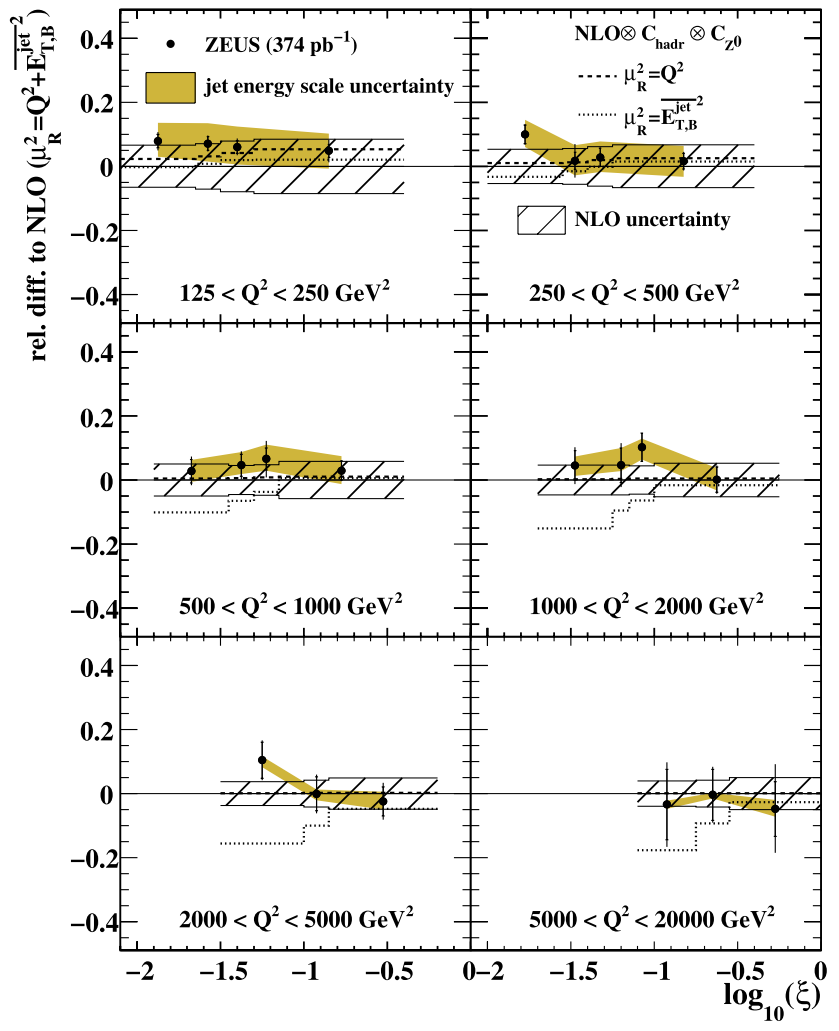


Fig. 13 The measured differential cross-section $d \sigma / d \overline{E_{T, \mathrm{~B}}^{\mathrm{jet}}}$ for inclusive dijet production in different regions of $Q^{2}$. Other details as in the caption to Fig. 8
Fig. 14 Relative differences between the measured differential cross-sections $d \sigma / d \overline{E_{T, \mathrm{~B}}^{\text {jet }}}$ presented in Fig. 13 and the NLO QCD calculations with $\mu_{R}^{2}=Q^{2}+{\overline{E_{T, \mathrm{~B}}^{\mathrm{jet}}}}^{2}$. Other details as in the caption to Fig. 8

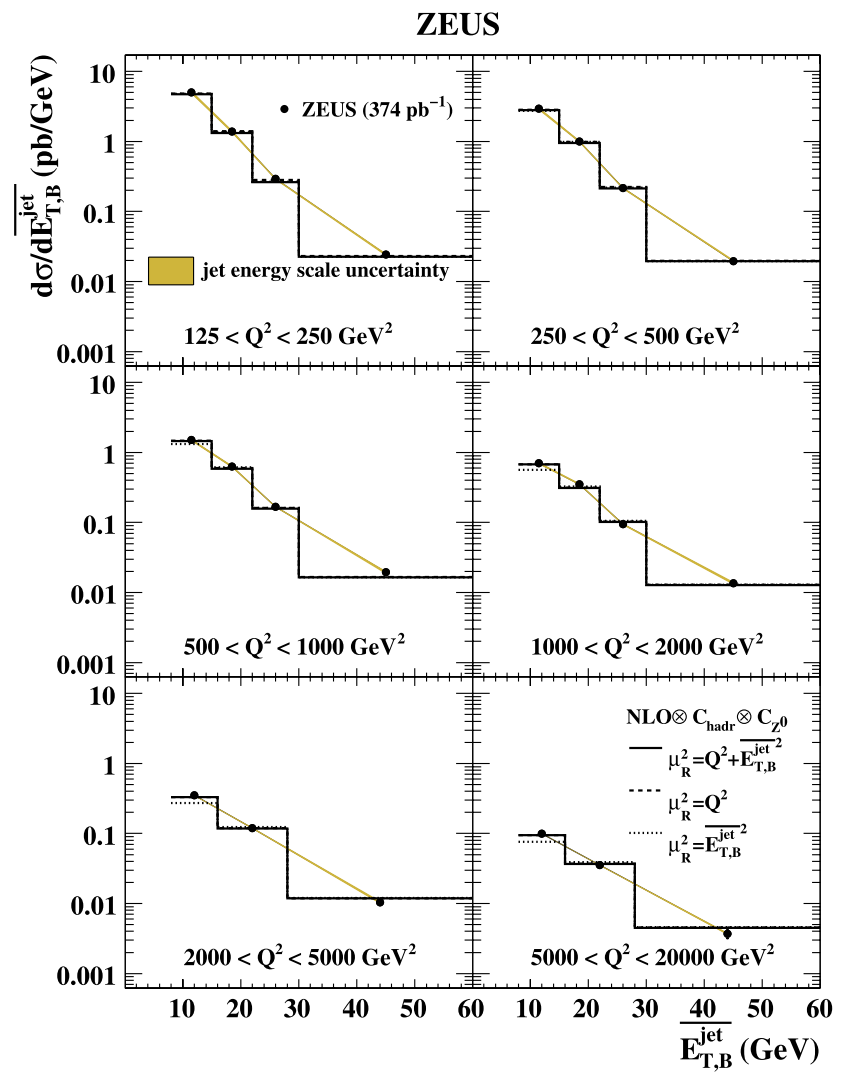

\section{ZEUS}

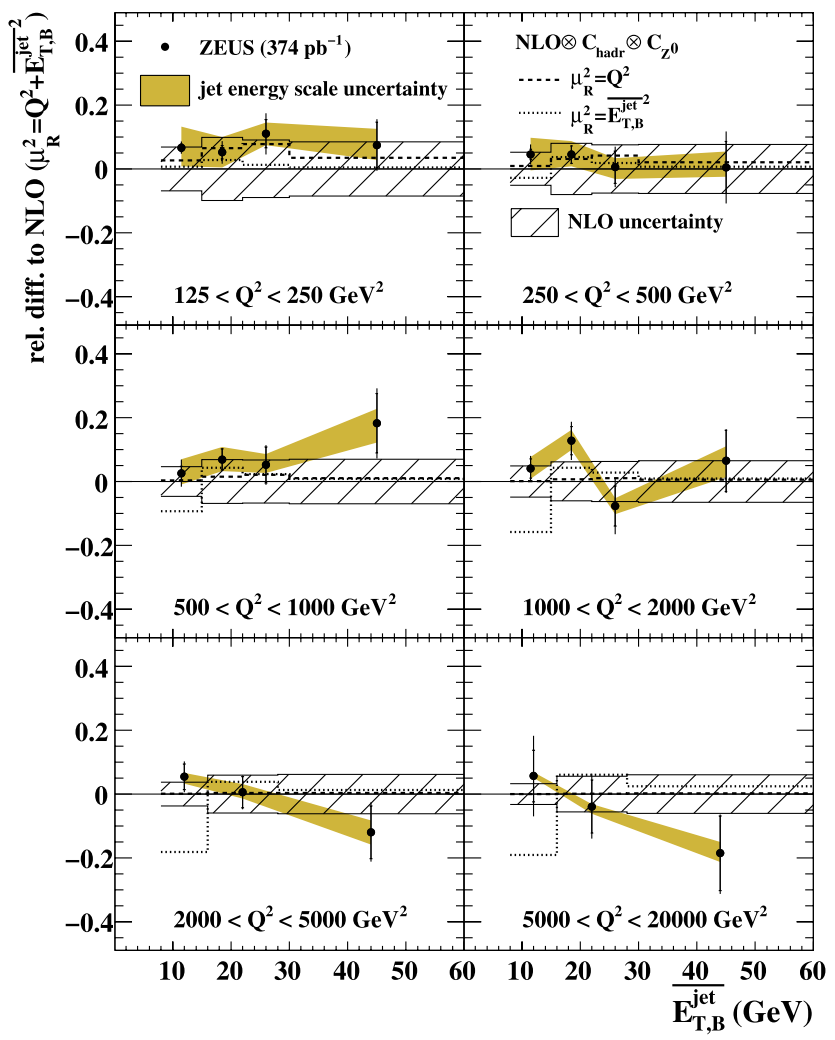


Table 7 Inclusive dijet cross-sections $d \sigma / d \log _{10}(\xi)$ in different regions of $Q^{2}$. Other details as in the caption to Table 1

\begin{tabular}{|c|c|c|c|c|c|c|}
\hline \multirow[t]{2}{*}{$\log _{10}(\xi)$ bin } & \multicolumn{4}{|c|}{$d \sigma / d \log _{10}(\xi)$} & \multirow[b]{2}{*}{$C_{\mathrm{QED}}$} & \multirow[b]{2}{*}{$C_{\text {hadr }} \cdot C_{\mathrm{Z}}$} \\
\hline & $(\mathrm{pb})$ & $\delta_{\text {stat }}$ & $\delta_{\text {syst }}$ & $\delta_{\mathrm{ES}}$ & & \\
\hline \multicolumn{7}{|c|}{$125<Q^{2}<250 \mathrm{GeV}^{2}$} \\
\hline$-2.1-(-1.65)$ & 30.34 & \pm 0.60 & ${ }_{-0.55}^{+0.51}$ & $\begin{array}{l}+1.58 \\
-1.38\end{array}$ & 0.97 & 0.95 \\
\hline$-1.65-(-1.5)$ & 76.2 & \pm 1.5 & $\begin{array}{l}+1.0 \\
-0.9\end{array}$ & $\begin{array}{l}+4.5 \\
-4.0\end{array}$ & 0.96 & 0.94 \\
\hline$-1.5-(-1.3)$ & 63.2 & \pm 1.2 & $\begin{array}{l}+1.1 \\
-1.0\end{array}$ & $\begin{array}{l}+3.8 \\
-3.3\end{array}$ & 0.97 & 0.97 \\
\hline$-1.3-(-0.4)$ & 11.53 & \pm 0.22 & ${ }_{-0.33}^{+0.31}$ & $\begin{array}{l}+0.60 \\
-0.61\end{array}$ & 0.97 & 0.94 \\
\hline \multicolumn{7}{|c|}{$250<Q^{2}<500 \mathrm{GeV}^{2}$} \\
\hline$-2-(-1.55)$ & 19.93 & \pm 0.52 & $\begin{array}{l}+0.29 \\
-0.27\end{array}$ & $\begin{array}{l}+0.82 \\
-0.71\end{array}$ & 0.96 & 0.95 \\
\hline$-1.55-(-1.4)$ & 48.2 & \pm 1.3 & $\begin{array}{l}+2.1 \\
-2.1\end{array}$ & $\begin{array}{l}+2.3 \\
-2.2\end{array}$ & 0.94 & 0.96 \\
\hline$-1.4-(-1.25)$ & 42.8 & \pm 1.2 & $\begin{array}{l}+0.5 \\
-0.4\end{array}$ & $\begin{array}{l}+2.1 \\
-1.9\end{array}$ & 0.95 & 0.96 \\
\hline$-1.25-(-0.4)$ & 8.56 & \pm 0.21 & $\begin{array}{l}+0.13 \\
-0.14\end{array}$ & $\begin{array}{l}+0.40 \\
-0.41\end{array}$ & 0.95 & 0.95 \\
\hline \multicolumn{7}{|c|}{$500<Q^{2}<1000 \mathrm{GeV}^{2}$} \\
\hline$-1.9-(-1.45)$ & 8.21 & \pm 0.29 & $\begin{array}{l}+0.22 \\
-0.19\end{array}$ & $\begin{array}{l}+0.29 \\
-0.27\end{array}$ & 0.94 & 0.94 \\
\hline$-1.45-(-1.3)$ & 28.32 & \pm 0.93 & $\begin{array}{l}+0.74 \\
-0.72\end{array}$ & $\begin{array}{l}+1.10 \\
-0.76\end{array}$ & 0.94 & 0.96 \\
\hline$-1.3-(-1.15)$ & 29.11 & \pm 0.93 & $\begin{array}{l}+1.23 \\
-1.27\end{array}$ & $\begin{array}{l}+1.22 \\
-1.02\end{array}$ & 0.94 & 0.97 \\
\hline$-1.15-(-0.4)$ & 6.00 & \pm 0.17 & ${ }_{-0.08}^{+0.07}$ & ${ }_{-0.25}^{+0.26}$ & 0.95 & 0.97 \\
\hline \multicolumn{7}{|c|}{$1000<Q^{2}<2000 \mathrm{GeV}^{2}$} \\
\hline$-1.7-(-1.25)$ & 4.91 & \pm 0.22 & $\begin{array}{l}+0.16 \\
-0.16\end{array}$ & ${ }_{-0.15}^{+0.13}$ & 0.94 & 0.95 \\
\hline$-1.25-(-1.15)$ & 15.52 & \pm 0.80 & $\begin{array}{l}+0.64 \\
-0.63\end{array}$ & $\begin{array}{l}+0.80 \\
-0.27\end{array}$ & 0.93 & 0.98 \\
\hline$-1.15-(-1)$ & 16.87 & \pm 0.68 & $\begin{array}{l}+0.10 \\
-0.15\end{array}$ & $\begin{array}{l}+0.41 \\
-0.58\end{array}$ & 0.94 & 0.98 \\
\hline$-1-(-0.25)$ & 2.97 & \pm 0.12 & $\begin{array}{l}+0.06 \\
-0.06\end{array}$ & ${ }_{-0.10}^{+0.11}$ & 0.93 & 1.00 \\
\hline \multicolumn{7}{|c|}{$2000<Q^{2}<5000 \mathrm{GeV}^{2}$} \\
\hline$-1.5-(-1)$ & 3.11 & \pm 0.16 & $\begin{array}{l}+0.07 \\
-0.08\end{array}$ & ${ }_{-0.06}^{+0.04}$ & 0.93 & 1.03 \\
\hline$-1-(-0.85)$ & 9.07 & \pm 0.48 & $\begin{array}{l}+0.26 \\
-0.28\end{array}$ & ${ }_{-0.18}^{+0.12}$ & 0.92 & 1.03 \\
\hline$-0.85-(-0.2)$ & 2.54 & \pm 0.12 & $\begin{array}{l}+0.09 \\
-0.09\end{array}$ & $\begin{array}{l}+0.08 \\
-0.07\end{array}$ & 0.93 & 1.04 \\
\hline \multicolumn{7}{|c|}{$5000<Q^{2}<20000 \mathrm{GeV}^{2}$} \\
\hline$-1.1-(-0.75)$ & 0.865 & \pm 0.099 & $\begin{array}{l}+0.065 \\
-0.065\end{array}$ & ${ }_{-0.013}^{+0.011}$ & 0.94 & 1.10 \\
\hline$-0.75-(-0.55)$ & 2.85 & \pm 0.23 & $\begin{array}{l}+0.10 \\
-0.10\end{array}$ & $\begin{array}{l}+0.02 \\
-0.03\end{array}$ & 0.91 & 1.08 \\
\hline$-0.55-0$ & 0.794 & \pm 0.071 & $\begin{array}{l}+0.092 \\
-0.090\end{array}$ & $\begin{array}{l}+0.023 \\
-0.020\end{array}$ & 0.92 & 1.10 \\
\hline
\end{tabular}

they are of the order of 5 to $10 \%$, with the smaller values at higher $Q^{2}$. Data and theory are in good agreement over the whole measured range.

\section{Summary and conclusions}

Measurements of single- and double-differential cross sections for dijet production at high- $Q^{2}$ NC DIS were made using an integrated luminosity of $374 \mathrm{pb}^{-1}$. The measurements have very small statistical and systematic uncertainties and the description of the data by the predictions of NLO QCD is very good, giving a powerful and stringent justification of the theory. These data will provide useful precision information for the determination of the strong coupling constant and the extraction of the proton PDFs. 
Table 8 Inclusive dijet cross-sections $d \sigma / d \overline{E_{T, \mathrm{~B}}^{\mathrm{jet}}}$ in different regions of $Q^{2}$. Other details as in the caption to Table 1

\begin{tabular}{|c|c|c|c|c|c|c|}
\hline \multirow{2}{*}{$\begin{array}{l}\overline{E_{T, \mathrm{~B}}^{\mathrm{jet}}} \text { bin } \\
(\mathrm{GeV})\end{array}$} & \multicolumn{4}{|l|}{$d \sigma / \overline{E_{T, \mathrm{~B}}^{\mathrm{jet}}}$} & \multirow[b]{2}{*}{$C_{\mathrm{QED}}$} & \multirow[b]{2}{*}{$C_{\text {hadr }} \cdot C_{Z^{0}}$} \\
\hline & $(\mathrm{pb} / \mathrm{GeV})$ & $\delta_{\text {stat }}$ & $\delta_{\text {syst }}$ & $\delta_{\mathrm{ES}}$ & & \\
\hline \multicolumn{7}{|c|}{$125<Q^{2}<250 \mathrm{GeV}^{2}$} \\
\hline $8-15$ & 5.050 & \pm 0.057 & $\begin{array}{l}+0.071 \\
-0.070\end{array}$ & $\begin{array}{l}+0.311 \\
-0.274\end{array}$ & 0.97 & 0.95 \\
\hline $15-22$ & 1.385 & \pm 0.028 & $\begin{array}{l}+0.037 \\
-0.038\end{array}$ & $\begin{array}{l}+0.063 \\
-0.062\end{array}$ & 0.97 & 0.96 \\
\hline $22-30$ & 0.292 & \pm 0.012 & $\begin{array}{l}+0.012 \\
-0.013\end{array}$ & $\begin{array}{l}+0.009 \\
-0.010\end{array}$ & 0.97 & 0.96 \\
\hline $30-60$ & 0.0241 & \pm 0.0016 & $\begin{array}{l}+0.0009 \\
-0.0008\end{array}$ & $\begin{array}{l}+0.0011 \\
-0.0010\end{array}$ & 0.97 & 0.95 \\
\hline \multicolumn{7}{|c|}{$250<Q^{2}<500 \mathrm{GeV}^{2}$} \\
\hline $8-15$ & 2.937 & \pm 0.046 & $\begin{array}{l}+0.076 \\
-0.076\end{array}$ & $\begin{array}{l}+0.146 \\
-0.141\end{array}$ & 0.95 & 0.95 \\
\hline $15-22$ & 0.998 & \pm 0.026 & ${ }_{-0.011}^{+0.011}$ & ${ }_{-0.035}^{+0.040}$ & 0.95 & 0.98 \\
\hline $22-30$ & 0.215 & \pm 0.011 & $\begin{array}{l}+0.008 \\
-0.008\end{array}$ & $\begin{array}{l}+0.006 \\
-0.008\end{array}$ & 0.96 & 0.97 \\
\hline $30-60$ & 0.0195 & \pm 0.0016 & $\begin{array}{l}+0.0015 \\
-0.0015\end{array}$ & $\begin{array}{l}+0.0010 \\
{ }_{-0} .0006\end{array}$ & 0.93 & 0.95 \\
\hline \multicolumn{7}{|c|}{$500<Q^{2}<1000 \mathrm{GeV}^{2}$} \\
\hline $8-15$ & 1.502 & \pm 0.031 & $\begin{array}{l}+0.055 \\
-0.054\end{array}$ & $\begin{array}{l}+0.064 \\
-0.052\end{array}$ & 0.94 & 0.95 \\
\hline $15-22$ & 0.629 & \pm 0.019 & $\begin{array}{l}+0.008 \\
-0.009\end{array}$ & $\begin{array}{l}+0.023 \\
-0.021\end{array}$ & 0.95 & 0.99 \\
\hline $22-30$ & 0.1665 & \pm 0.0089 & ${ }_{-0.0041}^{+0.0041}$ & $\begin{array}{l}+0.0054 \\
-0.0040\end{array}$ & 0.96 & 0.98 \\
\hline $30-60$ & 0.0194 & \pm 0.0015 & $\begin{array}{l}+0.0010 \\
-0.0010\end{array}$ & $\begin{array}{l}+0.0007 \\
-0.0010\end{array}$ & 0.95 & 0.99 \\
\hline \multicolumn{7}{|c|}{$1000<Q^{2}<2000 \mathrm{GeV}^{2}$} \\
\hline $8-15$ & 0.701 & \pm 0.020 & $\begin{array}{l}+0.017 \\
-0.017\end{array}$ & $\begin{array}{l}+0.025 \\
-0.022\end{array}$ & 0.93 & 0.95 \\
\hline $15-22$ & 0.352 & \pm 0.014 & ${ }_{-0.013}^{+0.012}$ & $\begin{array}{l}+0.011 \\
-0.009\end{array}$ & 0.94 & 1.01 \\
\hline $22-30$ & 0.0943 & \pm 0.0064 & $\begin{array}{l}+0.0063 \\
-0.0063\end{array}$ & $\begin{array}{l}+0.0025 \\
-0.0026\end{array}$ & 0.94 & 1.02 \\
\hline $30-60$ & 0.0136 & \pm 0.0012 & $\begin{array}{l}+0.0003 \\
-0.0004\end{array}$ & $\begin{array}{l}+0.0006 \\
-0.0007\end{array}$ & 0.94 & 1.04 \\
\hline \multicolumn{7}{|c|}{$2000<Q^{2}<5000 \mathrm{GeV}^{2}$} \\
\hline $8-16$ & 0.350 & \pm 0.013 & $\begin{array}{l}+0.009 \\
-0.009\end{array}$ & $\begin{array}{l}+0.004 \\
-0.007\end{array}$ & 0.92 & 1.00 \\
\hline $16-28$ & 0.1191 & \pm 0.0058 & $\begin{array}{l}+0.0023 \\
-0.0022\end{array}$ & $\begin{array}{l}+0.0030 \\
-0.0023\end{array}$ & 0.93 & 1.07 \\
\hline $28-60$ & 0.01040 & \pm 0.00097 & $\begin{array}{l}+0.00053 \\
-0.00049\end{array}$ & $\begin{array}{l}+0.00044 \\
-0.00046\end{array}$ & 0.94 & 1.08 \\
\hline \multicolumn{7}{|c|}{$5000<Q^{2}<20000 \mathrm{GeV}^{2}$} \\
\hline $8-16$ & 0.0995 & \pm 0.0076 & $\begin{array}{l}+0.0092 \\
{ }_{-0.0092}\end{array}$ & $\begin{array}{l}+0.0012 \\
-0.0005\end{array}$ & 0.93 & 1.05 \\
\hline $16-28$ & 0.0354 & \pm 0.0031 & $\begin{array}{l}+0.0023 \\
-0.0021\end{array}$ & $\begin{array}{l}+0.0003 \\
-0.0008\end{array}$ & 0.89 & 1.14 \\
\hline $28-60$ & 0.00368 & \pm 0.00053 & $\begin{array}{l}+0.00015 \\
-0.00023 \\
\end{array}$ & $\begin{array}{l}+0.00016 \\
-0.00012 \\
\end{array}$ & 0.95 & 1.20 \\
\hline
\end{tabular}

Acknowledgements We thank the DESY Directorate for their strong support and encouragement. The remarkable achievements of the HERA machine group were essential for the successful completion of this work and are greatly appreciated. We are grateful for the support of the DESY computing and network services. The design, construction and installation of the ZEUS detector have been made possible owing to the ingenuity and effort of many people from DESY and home institutes who are not listed as authors. We would like to thank Z. Nagy for useful discussions.

Open Access This article is distributed under the terms of the Creative Commons Attribution Noncommercial License which permits any noncommercial use, distribution, and reproduction in any medium, provided the original author(s) and source are credited.

\section{References}

1. C. Adloff et al. (H1 Collaboration), Eur. Phys. J. C 19, 289 (2001)

2. J. Breitweg et al. (ZEUS Collaboration), Phys. Lett. B 507, 70 (2001)

3. S. Chekanov et al. (ZEUS Collaboration), Phys. Lett. B 547, 164 (2002)

4. S. Chekanov et al. (ZEUS Collaboration), Eur. Phys. J. C 23, 615 (2002)

5. S. Chekanov et al. (ZEUS Collaboration), Phys. Lett. B 560, 7 (2003)

6. A. Aktas et al. (H1 Collaboration), Phys. Lett. B 653, 134 (2007)

7. S. Chekanov et al. (ZEUS Collaboration), Nucl. Phys. B 765, 1 (2007) 
8. S. Chekanov et al. (ZEUS Collaboration), Phys. Lett. B 649, 12 (2007)

9. F.D. Aaron et al. (H1 Collaboration), Eur. Phys. J. C 65, 363 (2010)

10. H. Abramowicz et al. (ZEUS Collaboration), Phys. Lett. B 691, $127(2010)$

11. C. Adloff et al. (H1 Collaboration), Phys. Lett. B 542, 193 (2002)

12. C. Adloff et al. (H1 Collaboration), Eur. Phys. J. C 29, 497 (2003)

13. A. Aktas et al. (H1 Collaboration), Eur. Phys. J. C 33, 477 (2004)

14. S. Chekanov et al. (ZEUS Collaboration), Eur. Phys. J. C 44, 183 (2005)

15. S. Chekanov et al. (ZEUS Collaboration), Phys. Rev. D 76, 072011 (2007)

16. S. Chekanov et al. (ZEUS Collaboration), Phys. Rev. D 78, 032004 (2008)

17. S. Chekanov et al. (ZEUS Collaboration), Nucl. Phys. B 792, 1 (2008)

18. R.P. Feynman, Photon-Hadron Interactions (Benjamin, New York, 1972)

19. K.H. Streng, T.F. Walsh, P.M. Zerwas, Z. Phys. C 2, 237 (1979)

20. S. Chekanov et al. (ZEUS Collaboration), Eur. Phys. J. C 42, 1 (2005)

21. (ZEUS Collaboration) U. Holm (ed.), The ZEUS Detector. Status Report (unpublished) DESY, 1993, available on http://www-zeus.desy.de/bluebook/bluebook.html

22. N. Harnew et al., Nucl. Instrum. Methods Phys. Res. A 279, 290 (1989)

23. B. Foster et al., Nucl. Phys. B, Proc. Suppl. 32, 181 (1993)

24. B. Foster et al., Nucl. Instrum. Methods Phys. Res. A 338, 254 (1994)

25. A. Polini et al., Nucl. Instrum. Methods Phys. Res. A 581, 656 (2007)

26. M. Derrick et al., Nucl. Instrum. Methods Phys. Res. A 309, 77 (1991)

27. A. Andresen et al., Nucl. Instrum. Methods Phys. Res. A 309, 101 (1991)

28. A. Caldwell et al., Nucl. Instrum. Methods Phys. Res. A 321, 356 (1992)

29. A. Bernstein et al., Nucl. Instrum. Methods Phys. Res. A 336, 23 (1993)

30. J. Andruszków et al., Preprint DESY-92-066 DESY, 1992

31. M. Derrick et al. (ZEUS Coll.), Z. Phys. C 63, 391 (1994)

32. J. Andruszków et al., Acta Phys. Pol. B 32, 2025 (2001)

33. M. Helbich et al., Nucl. Instrum. Methods Phys. Res. A 565, 572 (2006)

34. A.A. Sokolov, I.M. Ternov, Sov. Phys. Dokl. 8, 1203 (1964)

35. V.N. Baier, V.A. Khoze, Sov. J. Nucl. Phys. 9, 238 (1969)

36. D.P. Barber et al., Nucl. Instrum. Methods Phys. Res. A 329, 79 (1993)

37. M. Beckmann et al., Nucl. Instrum. Methods Phys. Res. A 479, 334 (2002)

38. J. Breitweg et al. (ZEUS Collaboration), Eur. Phys. J. C 1, 109 (1998)

39. W.H. Smith, K. Tokushuku, L.W. Wiggers, in Proc. Computing in High-Energy Physics (CHEP), ed. by C. Verkerk, W. Wojcik Annecy, France, September 1992 (CERN, Geneva, 1992), p. 222. Also in preprint DESY 92-150B

40. P.D. Allfrey et al., Nucl. Instrum. Methods Phys. Res. A 580, 1257 (2007)

41. H. Abramowicz, A. Caldwell, R. Sinkus, Nucl. Instrum. Methods Phys. Res. A 365, 508 (1995)

42. R. Sinkus, T. Voss, Nucl. Instrum. Methods Phys. Res. A 391, 360 (1997)
43. S. Bentvelsen, J. Engelen, P. Kooijman, in Proc. of the Workshop on Physics at HERA, vol. 1, ed. by W. Buchmüller, G. Ingelman, Hamburg, Germany, DESY (1992), p. 23

44. K.C. Höger, in Proc. of the Workshop on Physics at HERA, vol. 1, ed. by W. Buchmüller, G. Ingelman, Hamburg, Germany, DESY (1992), p. 43

45. S. Catani et al., Nucl. Phys. B 406, 187 (1993)

46. S.D. Ellis, D.E. Soper, Phys. Rev. D 48, 3160 (1993)

47. J.E. Huth et al., in Research Directions for the Decade. Proc. of Summer Study on High Energy Physics, 1990, ed. by E.L. Berger (World Scientific, Singapore, 1992), p. 134. Also in preprint FERMILAB-CONF-90-249-E

48. S. Chekanov et al. (ZEUS Collaboration), Phys. Lett. B 558, 41 (2003)

49. S. Chekanov et al. (ZEUS Collaboration), Phys. Lett. B 531, 9 (2002)

50. R. Brun et al., GEANT3, Technical Report CERN-DD/EE/84-1 CERN, 1987

51. A. Kwiatkowski, H. Spiesberger, H.-J. Möhring, Comput. Phys. Commun. 69, 155 (1992)

52. H. Spiesberger, An event generator for $e p$ interactions at HERA including radiative processes (Version 4.6), 1996, available on http://www.desy.de/ hspiesb/heracles.html

53. K. Charchuła, G.A. Schuler, H. Spiesberger, Comput. Phys. Commun. 81, 381 (1994)

54. H. Spiesberger, HERACLES and DJANGOH: Event Generation for ep Interactions at HERA Including Radiative Processes, 1998, available on http://wwwthep.physik.uni-mainz.de/ hspiesb/ djangoh/djangoh.html

55. Y. Azimov et al., Phys. Lett. B 165, 147 (1985)

56. G. Gustafson, Phys. Lett. B 175, 453 (1986)

57. G. Gustafson, U. Pettersson, Nucl. Phys. B 306, 746 (1988)

58. B. Andersson et al., Z. Phys. C 43, 625 (1989)

59. L. Lönnblad, Comput. Phys. Commun. 71, 15 (1992)

60. L. Lönnblad, Z. Phys. C 65, 285 (1995)

61. G. Ingelman, A. Edin, J. Rathsman, Comput. Phys. Commun. 101, 108 (1997)

62. H.L. Lai et al., Eur. Phys. J. C 12, 375 (2000)

63. B. Andersson et al., Phys. Rep. 97, 31 (1983)

64. T. Sjöstrand, Comput. Phys. Commun. 82, 74 (1994)

65. T. Sjöstrand, Comput. Phys. Commun. 39, 347 (1986)

66. T. Sjöstrand, M. Bengtsson, Comput. Phys. Commun. 43, 367 (1987)

67. T. Sjöstrand et al., Comput. Phys. Commun. 135, 238 (2001)

68. A. Arbuzov et al., Comput. Phys. Commun. 94, 128 (1996)

69. Z. Nagy, Z. Trocsanyi, Phys. Rev. Lett. 87, 082001 (2001)

70. P.M. Nadolsky et al., Phys. Rev. D 78, 013004 (2008)

71. S. Catani, M.H. Seymour, Nucl. Phys. B 485, 291 (1997). Erratum in Nucl. Phys. B 510, 503 (1998)

72. J. Behr, Ph.D. Thesis, DESY-THESIS-2010-038, Hamburg, 2010

73. A.D. Martin et al., Eur. Phys. J. C 63, 189 (2009)

74. S. Chekanov et al. (ZEUS Collaboration), Phys. Rev. D 67, 012007 (2003)

75. S. Bethke, Prog. Part. Nucl. Phys. 58, 351 (2007)

76. M. Wing (on behalf of the ZEUS Coll.), in Proc. of the 10th International Conference on Calorimetry in High Energy Physics, ed. by R. Zhu, Pasadena, USA (2002), p. 767. Also in preprint hep-ex/0206036

77. S. Chekanov et al. (ZEUS Coll.), Eur. Phys. J. C 21, 443 (2001)

78. S. Chekanov et al. (ZEUS Coll.), Eur. Phys. J. C 62, 625 (2009)

79. J. Breitweg et al. (ZEUS Coll.), Eur. Phys. J. C 11, 427 (1999) 